\title{
RNA-Seq based exploration of differentially expressed genes (DEGs) in cotton(Gossypium hirsutum. L) upon infection with Aspergillus tubingensis
}

Maria Khizar

Quaid-i-Azam University

Jianxin Shi

Shanghai Jiao Tong University

Urooj Haroon

Quaid-i-Azam University

Musrat Ali

Quaid-i-Azam University

Fiza Liaquat

Shanghai Jiao Tong University

Mahnoor Akbar

Quaid-i-Azam University

Hira Saleem

Quaid-i-Azam University

Asif Kamal

Quaid-i-Azam University

Kinza Minhas

Quaid-i-Azam University

Hassan Javed Chaudhary

Quaid-i-Azam University

Muhammad Farooq Hussain Munis ( $\square$ munis@qau.edu.pk)

Quaid-i-Azam University

\section{Research Article}

Keywords: RNA-Seq, DEGs, Cotton, Aspergillus tubingensis, MAPK

Posted Date: August 6th, 2021

DOI: https://doi.org/10.21203/rs.3.rs-770815/v1 
License: (c) (i) This work is licensed under a Creative Commons Attribution 4.0 International License. Read Full License 


\title{
RNA-Seq based exploration of differentially expressed genes (DEGs) in cotton (Gossypium hirsutum. L) upon infection with Aspergillus tubingensis
}

\author{
Maria Khizar \\ Department of Plant Sciences, Faculty of Biological Sciences, Quaid-i-Azam University, \\ Islamabad, 45320, Pakistan
}

\section{Jianxin Shi}

Joint International Research Laboratory of Metabolic and Developmental Sciences, School of Life Sciences and Biotechnology, Shanghai Jiao Tong University, Shanghai, China

\section{Urooj Haroon}

Department of Plant Sciences, Faculty of Biological Sciences, Quaid-i-Azam University, Islamabad, 45320, Pakistan

\section{Musrat Ali \\ Department of Plant Sciences, Faculty of Biological Sciences, Quaid-i-Azam University, Islamabad, 45320, Pakistan}

\section{Fiza Liaquat}

School of Agriculture and Biology, Shanghai Jiao Tong University, Shanghai, China

\author{
Mahnoor Akbar \\ Department of Plant Sciences, Faculty of Biological Sciences, Quaid-i-Azam University, \\ Islamabad, 45320, Pakistan
}

\section{Hira Saleem}

Department of Plant Sciences, Faculty of Biological Sciences, Quaid-i-Azam University, Islamabad, 45320, Pakistan 


\section{Asif Kamal}

Department of Plant Sciences, Faculty of Biological Sciences, Quaid-i-Azam University, Islamabad, 45320, Pakistan

\section{Kinza Minhas}

Department of Plant Sciences, Faculty of Biological Sciences, Quaid-i-Azam University, Islamabad, 45320, Pakistan

\section{Hassan Javed Chaudhary}

Department of Plant Sciences, Faculty of Biological Sciences, Quaid-i-Azam University, Islamabad, 45320, Pakistan

* Muhammad Farooq Hussain Munis (Corresponding Author)

Department of Plant Sciences, Faculty of Biological Sciences, Quaid-i-Azam University, Islamabad, 45320, Pakistan

Email addresses: munis@qau.edu.pk 


\title{
RNA-Seq based exploration of differentially expressed genes (DEGs) in cotton (Gossypium hirsutum. L) upon infection with Aspergillus tubingensis
}

\author{
Maria Khizar', Jianxin Shi ${ }^{2}$, Urooj Haroon', Musrat Ali, ${ }^{1}$, Fiza Liaquat ${ }^{3}$, Mahnoor Akbar1 \\ Hira Saleem¹, Asif Kamal ${ }^{1}$, Kinza Minhas' ${ }^{1}$, Hassan Javed Chaudhary ${ }^{1}$, Muhammad Farooq \\ Hussain Munis ${ }^{1 *}$ \\ ${ }^{1}$ Department of Plant Sciences, Faculty of Biological Sciences, Quaid-i-Azam University, \\ Islamabad, 45320, Pakistan \\ 2 Joint International Research Laboratory of Metabolic and Developmental Sciences, School of \\ Life Sciences and Biotechnology, Shanghai Jiao Tong University, Shanghai, China \\ ${ }^{3}$ School of Agriculture and Biology, Shanghai Jiao Tong University, Shanghai, China \\ * Corresponding Author: Muhammad Farooq Hussain Munis \\ Department of Plant Sciences, Faculty of Biological Sciences, Quaid-i-Azam University, \\ Islamabad, 45320, Pakistan \\ Email addresses: munis@ qau.edu.pk
}

\begin{abstract}
Differentially expressed genes help in exploring plant defense mechanism under variable stress conditions. In current investigation, RNA sequencing was executed to explore the differential gene expression in resistant and susceptible varieties of Cotton (Gossypium hirsutum), upon infection with Aspergillus tubingensis. Comparative RNA-Seq of control and infected plants was performed using Illumina HiSeq 2,500. Overall 79.84 G clean data was generated and 6,558 DEGs were identified in both varieties, in response to pathogen inoculation. Differentially expressed genes were found to be involved in defense, antifungal response, signaling pathways, oxidative burst and transcription. Genes involved in defense responses, MAPK signaling, cell wall fortification and signal transduction were highly induced in resistant variety. Real time PCR also revealed the up regulation of MAPKKK YODA like, L-ascorbate oxidase, late embryogenesis abundant protein (At1g64065) and flavonoid 3',5'-hydroxylase-like, in resistant variety. Elevated accumulation of such DEGs in resistant variety could function as the source for identifying biomarkers for breeding
\end{abstract}


and these can be used as potential candidate genes for transgenic manipulation. Their study also helped in understanding complex plant-fungal interaction and advanced the understanding of plantmicrobe interaction. Inclusively, our findings provide an indispensable foundation for advanced understanding of the plant resistance mechanisms of cotton.

Keywords: RNA-Seq, DEGs, Cotton, Aspergillus tubingensis, MAPK

\section{Introduction}

Cotton is considered as one of the world's most treasured crops. It provides a significant part of planet's regular fiber to the international yarn production. Cotton fiber signifies about $90 \%$ of entire cotton economic worth, while further economic value is secured from seed and other related items. China, United States of America and India produce the greater part of the world's cotton. In year 2011, a joined aggregate of more than 15.9 million metric tons of cotton fiber and 30.4 million metric tons of cottonseed, with an estimation of 22.8 billion dollars and 6 billion dollars, respectively was reported ${ }^{1}$. Upland cotton is a characteristic allotetraploid species that rose after interspecific hybridization among diploid species G. arboreum (A-like genome) and $G$. raimondii (D-like genome) ${ }^{2}$.

With the developments in the field of transcriptomics, RNA-sequencing has emerged as a major “-omics" technology for reviewing inclusive expression of genes under various stresses ${ }^{3}$. In cotton, stress mechanism had been studied under several biotic and abiotic factors. Currently, in Pakistan, transcriptomic analysis of G. hirsutum was carried out to investigate the DEGs in susceptible G. hirsutum variety in response to whiteflies attack ${ }^{4}$. RNA-seq analysis of infested cotton floral buds has exhibited the differential expression of plant hormone signaling genes, transcription factors and kinase cascades ${ }^{5}$. RNA-seq of $G$. hirsutum root tissues has also displayed differential genes expression in response to drought condition ${ }^{1}$. Comparative transcriptomic and reverse genetics have been employed for the identification of differentially expressed genes under the stress of Verticillium dahliae ${ }^{6}$. RNA seq analysis divulges variances in the mechanisms of origination and elongation among long- fiber and short-fiber cotton lines ${ }^{7}$. Transcriptomic analysis of $G$. hirsutum has also been applied for the detection of candidate genes in response to Aspergillus flavus $^{8}$.

Innate immune system of plants comprises of cells population and signaling pathways which constitutively function to provide quick response to pathogens, at diseased area ${ }^{9}$. Both pathogen triggered immunity and effector triggered immunity respond to stress by using a joint set 
of signaling components, which includes $\mathrm{ROS}, \mathrm{Ca}^{2+}$ and multiple protein kinases. These signaling machineries regulate downstream regulatory protein activities including transcription factors, which then lead to vast transcriptional reprogramming and initiates the induction and accretion of various pathogenesis-related proteins, enzymes and pathogen infection-responsive metabolites ${ }^{10}$. Rapid phosphorylation response against pathogens also involves some downstream mitogenactivated protein kinases (MAPKs) ${ }^{11}$ and calmodulin protein kinases ${ }^{12}$. Phytohormones play a central role in plant defense against pathogens. In A. thaliana, salicylic acid (SA) and ethylene can stimulate the expression of defensing-like (DEFL) genes ${ }^{13}$. The phenylpropanoid pathway plays a central role in plant defense against $V$. dahliae ${ }^{14}$. Furthermore, transcription factors such as ethylene response factors (ERFs) and WRKYs can be triggered by MAPKs, and are involved in

modulating plant defense ${ }^{15}$. A. tubingensis has been reported as a causal agent of leaf spot of cotton in Pakistan ${ }^{16}$. Non-targeted metabolomics profiling of G. hirsutum infected with A. tubingensis has depicted ample accumulation of significant metabolites like carbohydrates, flavonoids, fatty acids, phenylpropanoids organic acids, terpenoids and fatty acids in tolerant cultivar ${ }^{17}$.

This study was aimed to see the differential expression of different genes in tetraploid cotton, in response to A. tubingensis inoculation. Infected cotton cultivars were subjected to RNAseq analysis and obtained sequences were subjected to data analysis, gene ontology, functional annotation and pathway enrichment analysis. We intended to distinguish key DEGs of cotton in response to Aspergillus infection to interpret its resistance mechanisms.

\section{Results}

\section{Disease severity analysis}

Infected cotton leaves showed symptoms at 4 dpi. Leaves of susceptible variety exhibited infection spots, which later spread on the whole leaf surface. Initial symptoms appeared on leaf veins. The tolerant variety showed reduced leaf spots ( $2 \mathrm{~mm}$ average size) which did not spread rapidly (Fig.1A, B). Diseased area of fungal inoculated leaves of both varieties was measured in $\mathrm{cm}$ (Fig.1C). 
Figure 1
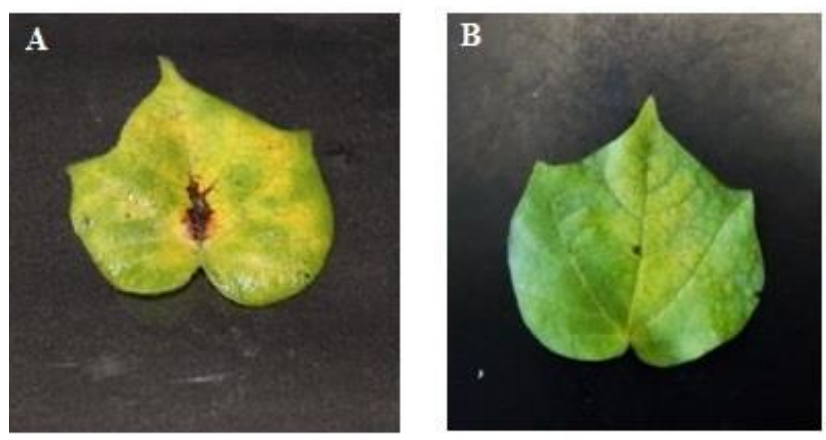

C

Disease Severity

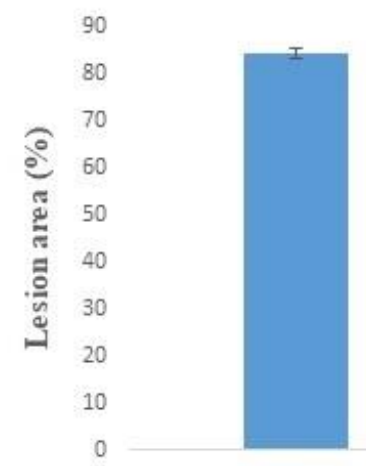

CIM-573
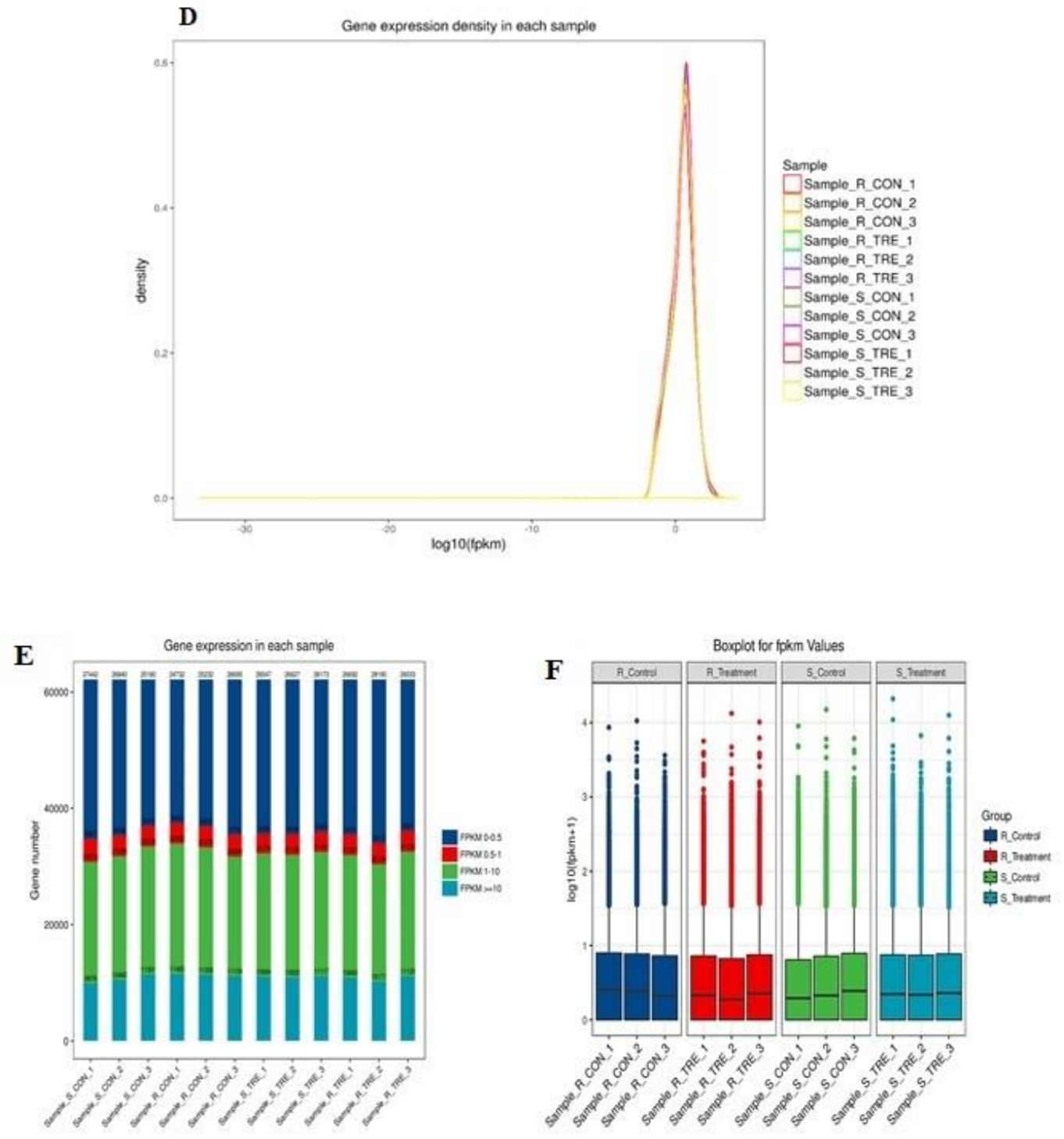

Figure 1. Phenotype of susceptible (A) and resistant cotton cultivar (B), after 4 days of infection with A. tubingensis. (C) Disease severity of susceptible and tolerant cultivars. (D) FPKM density 
distribution reflects the protein-coding gene expression pattern of each sample. (E) The FPKM value box line (F) FPKM box-plot for each sample. The X-axis is the sample name, Y-axis is $\log 10$ $(\mathrm{fpkm}+1)$. The different colors in the figure represent different ranges of fpkm values, the abscissa is the sample, and the ordinate is the number of protein-coding genes.

\section{Illumina Sequencing and Quality Control}

The complete analysis was comprised of 12 transcriptomic sequencing samples and a total of $79.84 \mathrm{G}$ clean data was obtained. The effective data volume of each sample was $6.19 \sim 6.82 \mathrm{G}$ with 44.37\% average GC content. The Q30 base distribution was found to be 91.62 93.46\% (Table 1).

Table 1. Preprocessing results of sequenced data quality

\begin{tabular}{|c|c|c|c|c|c|c|c|}
\hline $\begin{array}{l}\text { Sample } \\
\text { raw_reads }\end{array}$ & $\begin{array}{l}* \text { Raw } \\
\text { reads }(M)\end{array}$ & $\begin{array}{l}\text { Raw bases } \\
\text { (G) }\end{array}$ & $\begin{array}{l}\text { *Clean } \\
\text { reads }\end{array}$ & $\begin{array}{l}\text { Clea } \\
\text { base }\end{array}$ & $\begin{array}{l}\text { Valid } \\
\text { bases (\%) }\end{array}$ & $\begin{array}{l}\text { Q 30 } \\
(\%)\end{array}$ & $\begin{array}{l}\text { GC } \\
(\%)\end{array}$ \\
\hline $\begin{array}{l}\text { Sample_R_R } \\
\text { CON_1 }\end{array}$ & 49.90 & 7.49 & 47016742 & 6.74 & 90.08 & 91.88 & 44.13 \\
\hline $\begin{array}{l}\text { Sample_R_R } \\
\text { CON_2 }\end{array}$ & 49.42 & 7.41 & 46898960 & 6.71 & 90.54 & 92.43 & 44.54 \\
\hline $\begin{array}{l}\text { Sample_R_R } \\
\text { CON_3 }\end{array}$ & 46.11 & 6.92 & 43585100 & 6.19 & 89.48 & 91.87 & 44.12 \\
\hline $\begin{array}{l}\text { Sample_R_ } \\
\text { TRE_1 }\end{array}$ & 49.01 & 7.35 & 46053196 & 6.59 & 89.63 & 91.62 & 44.26 \\
\hline $\begin{array}{l}\text { Sample_R_ } \\
\text { TRE_2 }\end{array}$ & 49.39 & 7.41 & 46567842 & 6.66 & 89.94 & 92.13 & 44.22 \\
\hline $\begin{array}{l}\text { Sample_R_R } \\
\text { TRE_3 }\end{array}$ & 49.12 & 7.37 & 46329412 & 6.63 & 90.04 & 92.00 & 44.32 \\
\hline $\begin{array}{l}\text { Sample_S_ } \\
\text { CON_1 }\end{array}$ & 48.98 & 7.35 & 46575348 & 6.72 & 91.45 & 93.31 & 44.43 \\
\hline $\begin{array}{l}\text { Sample_S_ } \\
\text { CON_2 }\end{array}$ & 49.01 & 7.35 & 46730562 & 6.71 & 91.35 & 93.35 & 44.51 \\
\hline $\begin{array}{l}\text { Sample_S_ } \\
\text { CON_3 }\end{array}$ & 49.87 & 7.48 & 47505758 & 6.82 & 91.19 & 93.46 & 44.14 \\
\hline $\begin{array}{l}\text { Sample_S_T } \\
\text { RE_1 }\end{array}$ & 49.18 & 7.38 & 46478232 & 6.66 & 90.29 & 92.15 & 44.74 \\
\hline
\end{tabular}




\begin{tabular}{|l|l|l|l|l|l|l|l|l|}
\hline $\begin{array}{l}\text { Sample_S_T } \\
\text { RE_2 }\end{array}$ & 49.88 & 7.48 & 47342178 & 6.72 & & 89.88 & 92.42 & 44.81 \\
\hline $\begin{array}{l}\text { Sample_S_T } \\
\text { RE_3 }\end{array}$ & $49.35 \mathrm{M}$ & 7.40 & 46841020 & 6.69 & & 90.36 & 92.68 & 44.26 \\
\hline
\end{tabular}

Alignment to the Reference Genome

By comparing reads to the reference genome, the genomic alignment of each sample was obtained and the alignment rate was found to be $87.08 \sim 94.86 \%$. Based on the alignment results, proteincoding gene expression levels were successfully analyzed (Supplementary Table 1).

\section{FPKM density distribution}

The FPKM density distribution reflected protein-coding gene expression pattern of each sample (Fig.1D). A density map of FPKM for all sample genes visually represented the FPKM distribution of genes in each sample. Because there were differences in the number and value of gene expression, each sample expression value (FPKM) was distributed into different intervals. The number of genes expressed in different expression interval samples was calculated and the stacked histograms were drawn (Fig.1E)

In FPKM value Box-whisker plot, five statistics i.e. minimum, first quartile (25\%), median (50\%), third quartile (75\%) and maximum value were used to define information. It efficiently showed the symmetry of data and the degree of dispersion of distribution (Fig. 1F).

\section{Assessment of variation among samples}

PCA showed discrete behavior of resistant and susceptible variety and $74 \%$ total variance was contributed by all three PCs. PC1 showed $42.95 \%$ variance, while the variance of PC2 and PC3 was recorded to be $21.39 \%$ and $9.82 \%$, respectively (Fig. 2). Unsupervised hierarchical clustering of differentially expressed genes also provided useful information (Figure S1). Generally, the same types of samples were clustered together with similar biological functions. Constant with the prospects of the experimental conditions, pathogen infected samples in both varieties were clustered together. The closer was the correlation coefficient to 1; the elevated was the degree of similarity in expression patterns between samples (Figure S2). 


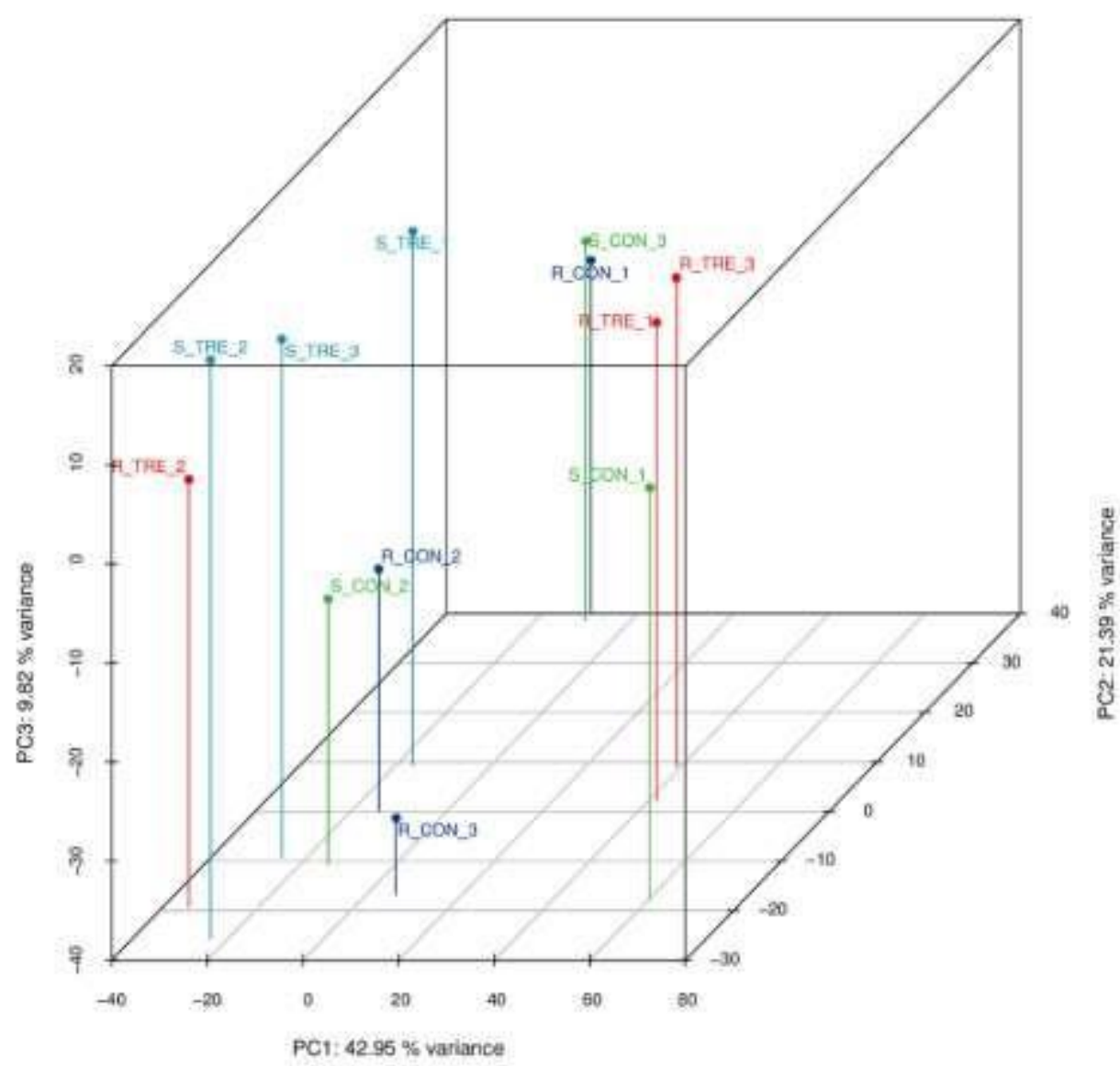

Figure 2.3D PCA exhibiting the variance of DEGs in all four data sets, with an overall variance of $74 \%$ contributed by all the three PC.

\section{Differential gene expression statistics and profiling}

According to the expression levels, protein-coding genes were found in four differential groups (Fig. 4). Numbers of differential protein-coding genes were detected to be 2,409 in group 1, 1998 in group 2, 302 in group 3 and 1,849 in group 4.

Based on the alignment of the obtained cotton reads with reference genome ( $G$. hirsutum), 6,558 DEGs was found in both varieties, using threshold of FC $>2$ and $\mathrm{P}<0.05$. In susceptible treated and susceptible control (ST vs SC) treatment, 1,070 genes were upregulated while 1,339 were down regulated. In resistant treated and resistant mock (RT vs RC) treatment, 849 genes were upregulated and 1,000 were down regulated. In resistant treated and susceptible treated condition (RT vs ST), 1,426 genes were upregulated and 572 genes were down regulated. On the other hand, in resistant control and susceptible control (RC vs SC), 217 genes were 
upregulated and 85 were suppressed, under similar conditions (Fig. 3A). Venn diagram showed common and unique DEGs in four data sets (Fig. 3B). Volcano plots also revealed the presence of up and down regulated genes among different treatments of resistant and susceptible varieties (Fig. 4).

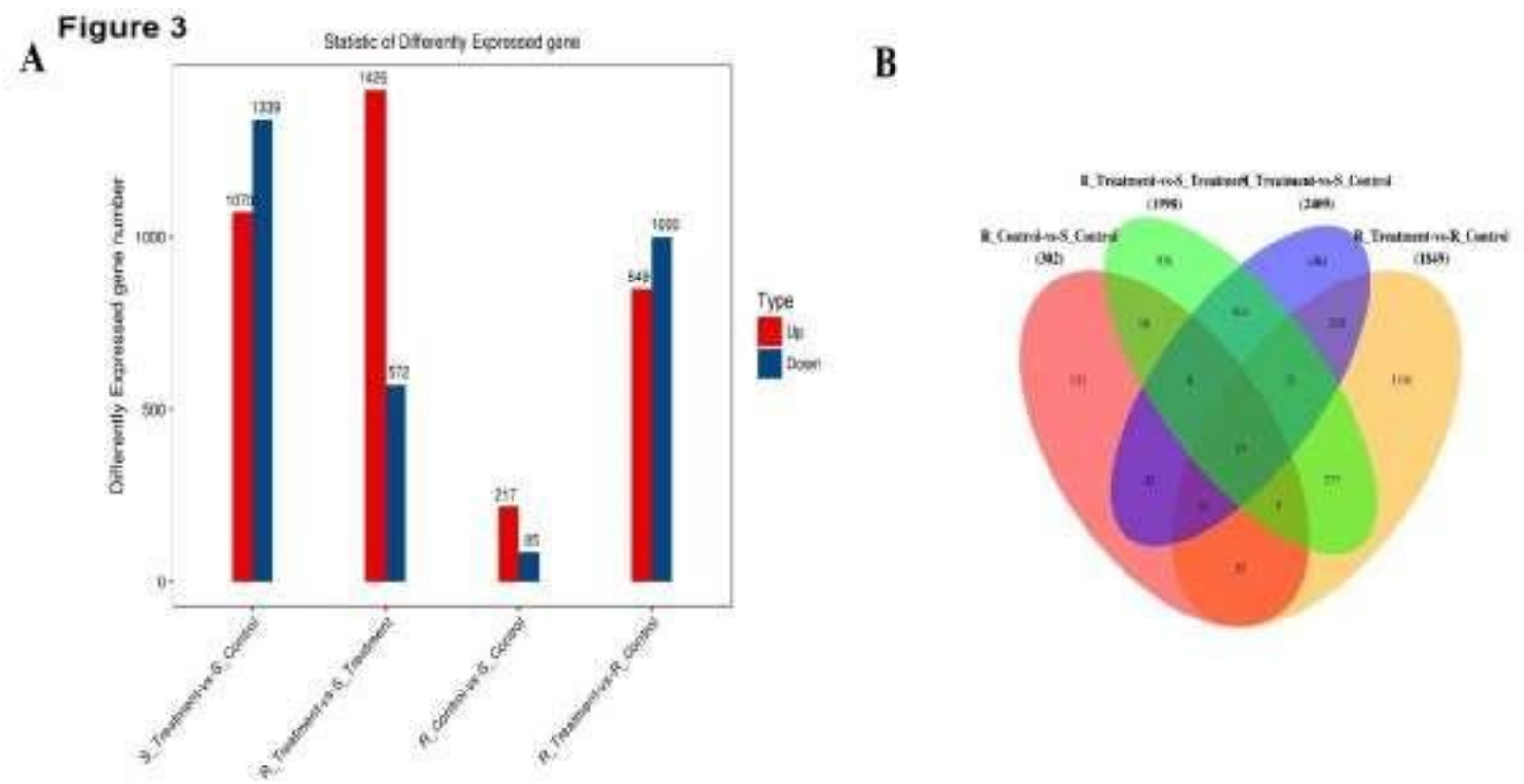

Figure 3. Statistical representation of differentially expressed genes horizontal .(A) Bar graph in which horizontal axis is the comparison group; the vertical axis is the number of differential genes in the comparison group, where "Up" is the number of up-regulated genes with significant differences, and "Down" is the number of down-regulated genes with significant differences. (B) Venn Graph is showing common and unique DEGs between different comparison groups. 


\section{Figure 4}

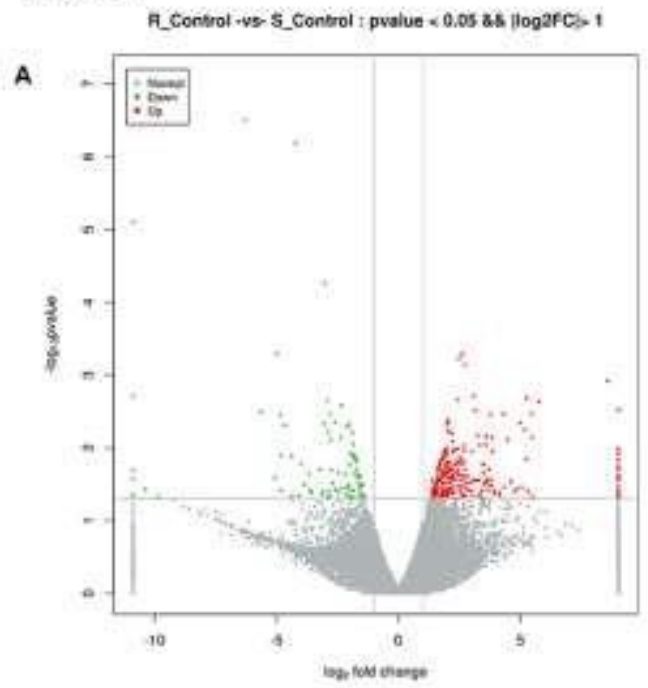

c

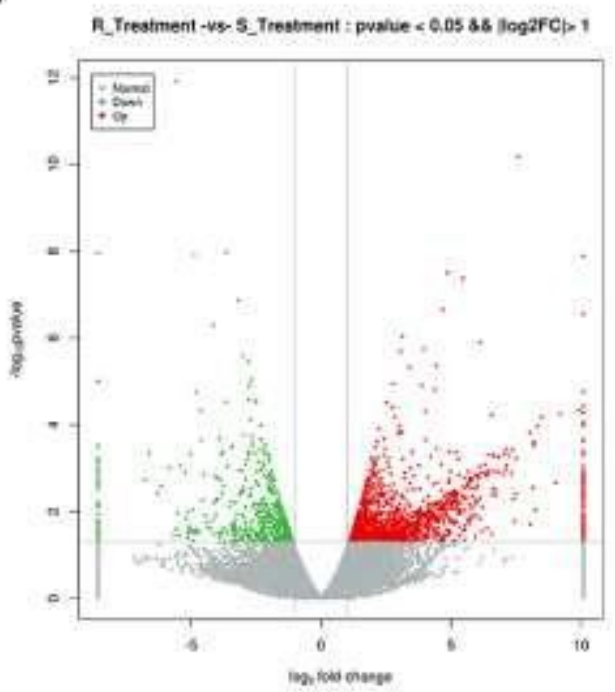

B

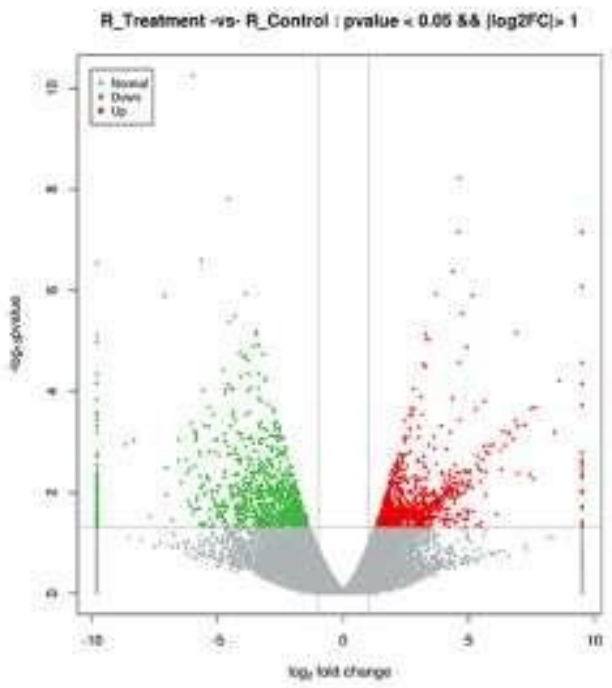

D

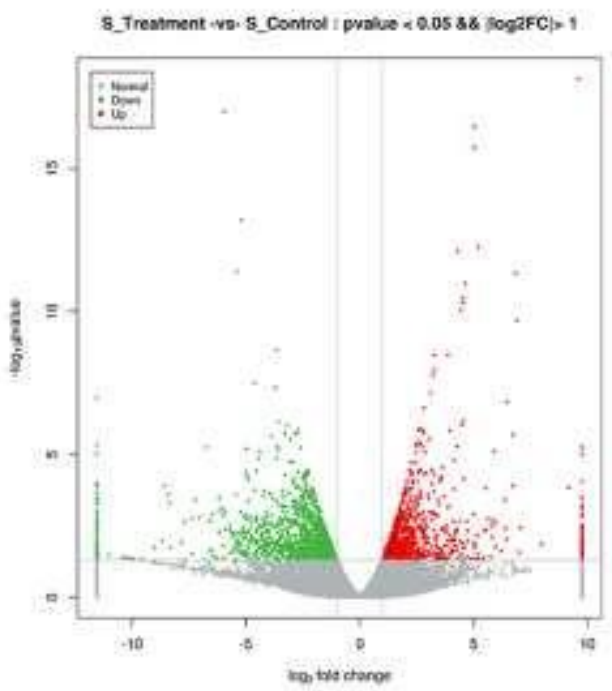

Figure 4. Differential expression by volcano map in four data sets. The gray color represents genes with a non-significant difference, while red and green are showing significantly up and down regulated genes. The horizontal axis is the display of $\log 2$ fold change, and the vertical axis is $-\log 10 \mathrm{P}$ value.

\section{Validation by Real Time PCR}

The transcriptomic data was confirmed using nine genes (with FC expression greater than 5), under inoculated conditions in both varieties. The results exhibited substantial up-regulation of mRNA, in response to A. tubingensis inoculation (Fig. 5). 


\section{Figure 5 Expression of Selected DEGs}

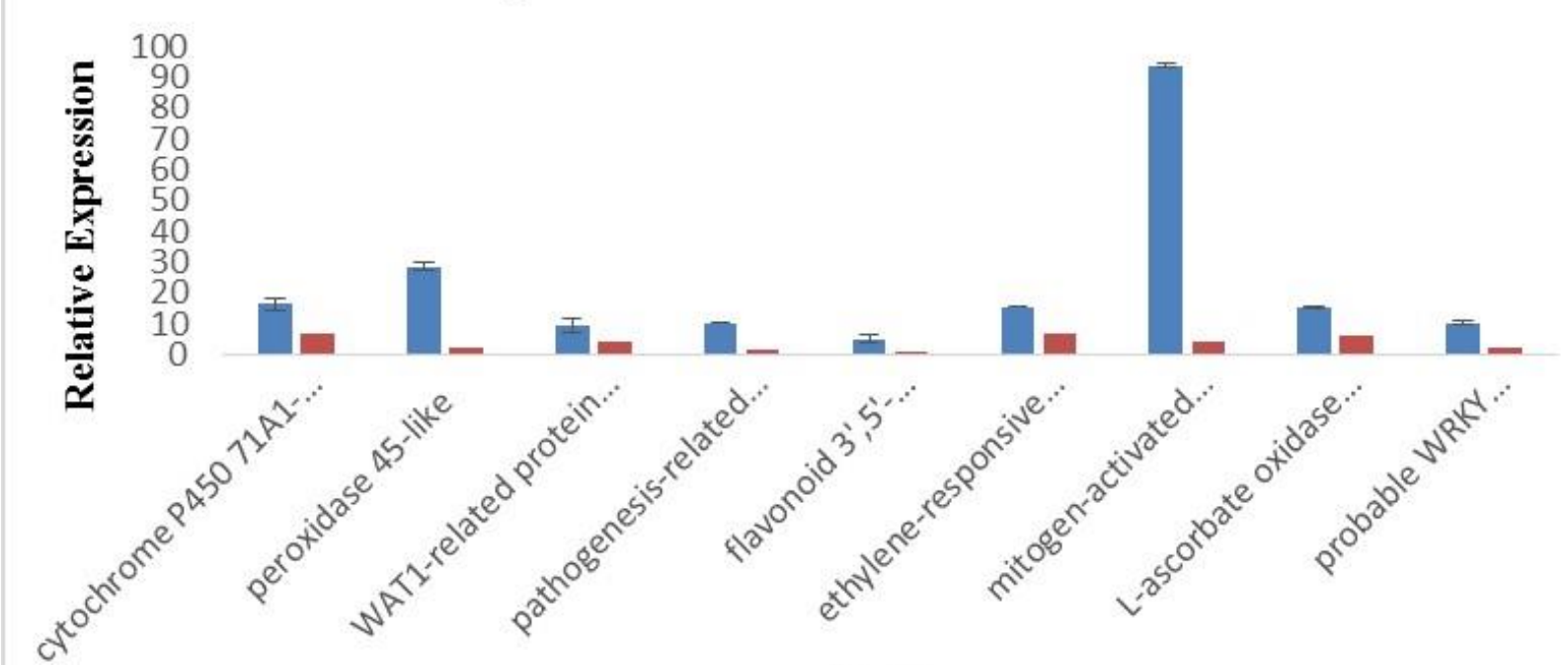

\section{DEGs}

RT $\square$ ST

Figure 5. Relative expressions of candidate genes (DEGs), in fungal inoculated resistant and susceptible varieties where $\mathrm{X}$-axis shows the relative expression of DEGs and Y-axis shows the genes.

\section{GO enrichment analysis of DEGs}

Based on gene ontology enrichment analysis, DEGs were distributed in different functional groups including $\mathrm{BP}, \mathrm{MF}$ and $\mathrm{CC}$ that were distinctly represented in resistant and susceptible variety in response to Aspergillus leaf spot. The DEGs were further distributed into 22, 13 and 13 sub-categories of BP, CC and MF, respectively, in resistant variety. DEGs were principally found to be involved in biological process including locomotion, signaling, response to stimulus, metabolic process and cellular process (Fig. 6A). The DEGs in susceptible cultivar post fungal inoculation were divided into 3 key subcategories, including biological process (15 DEGs), cellular process (13 DEGs) and molecular process (15 DEGs). The DEGs were mainly belonged to biological processes including localization, growth, biological regulation and cellular processes (Fig.6B). The DEGs for resistant and susceptible inoculated were presented in (Fig.6C). Detailed GO enrichment analysis of resistant cultivar (RT vs RC) has been provided in supplementary table (Table S2). 


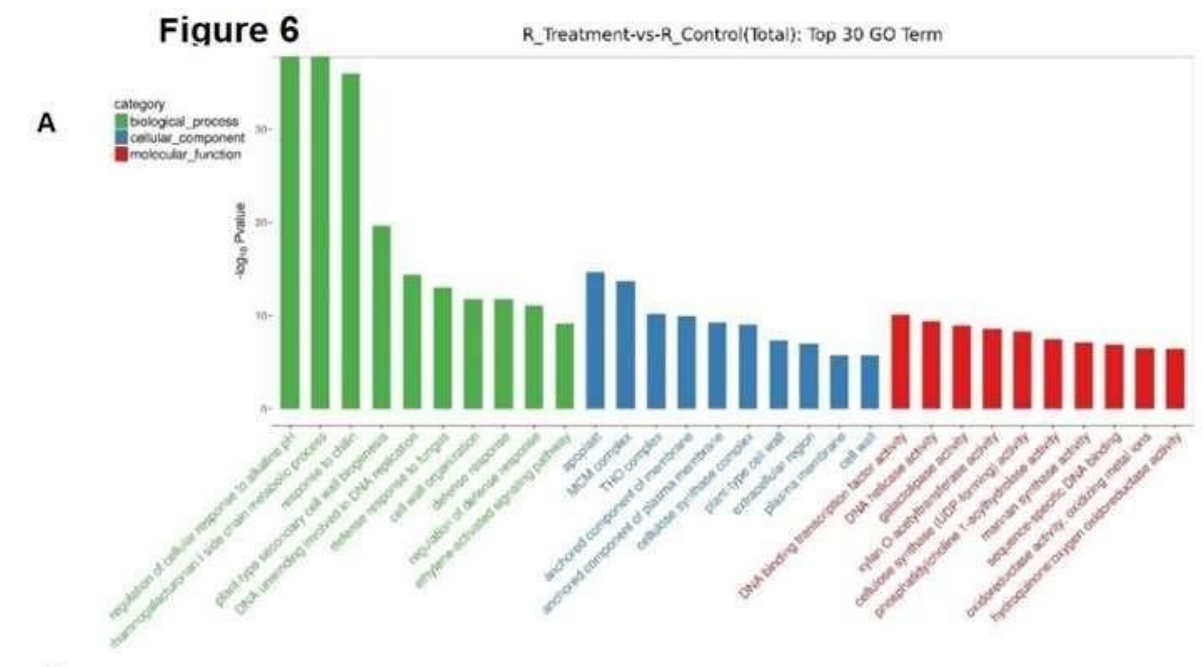

B

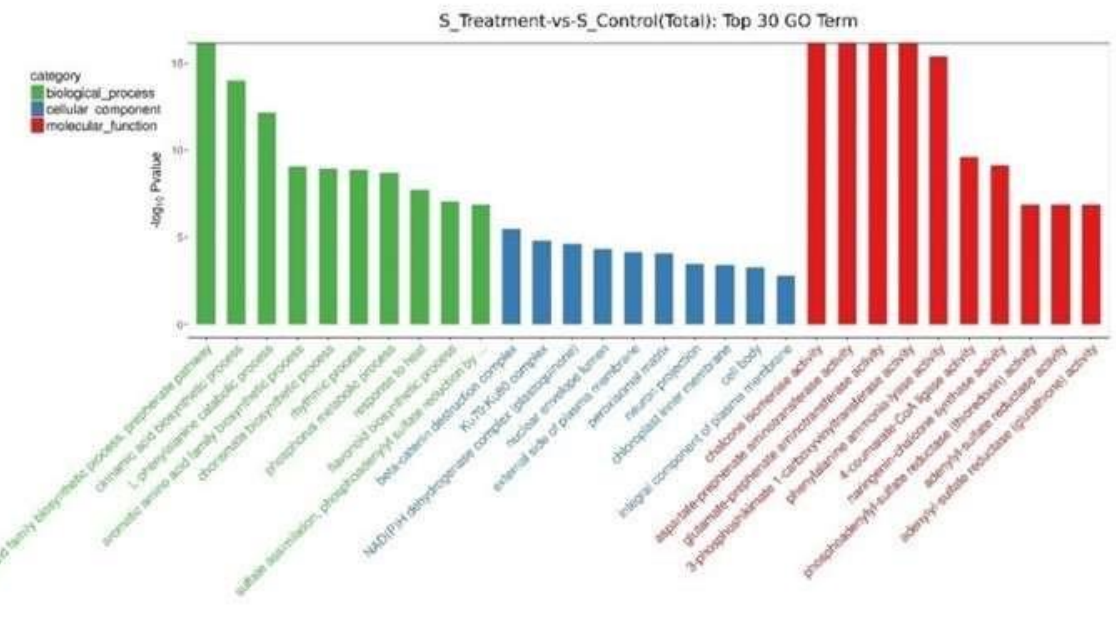

c

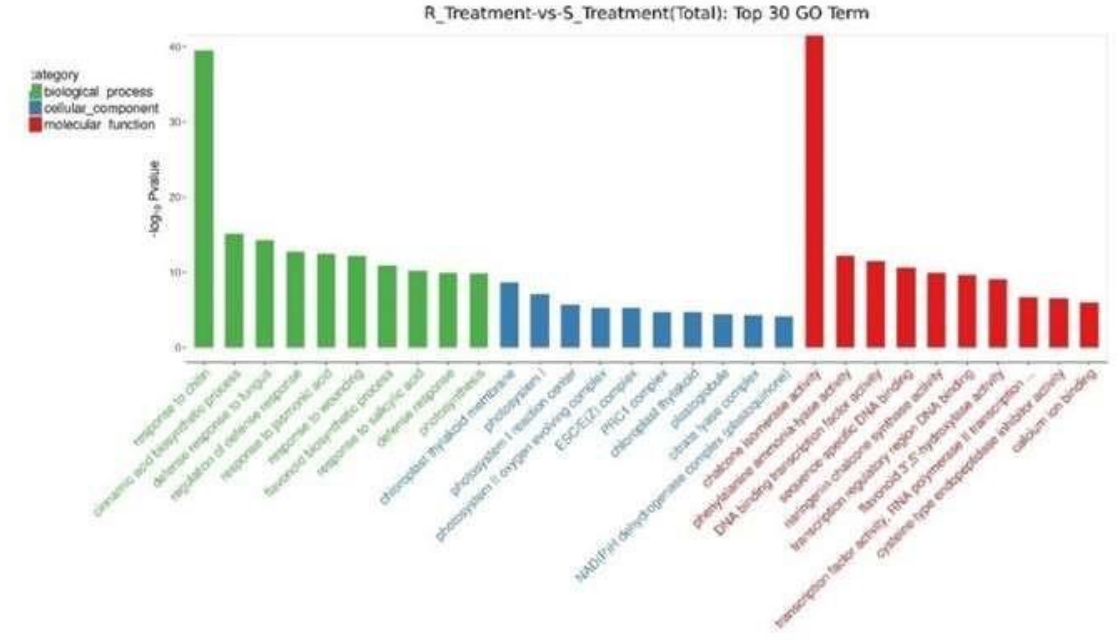

Figure.6 GO enrichment analysis of resistant variety (A), Susceptible variety (B) and 
Resistant pathogen inoculated and susceptible pathogen inoculated $(\mathbf{C})$ where green, blue and red color depicts biological, cellular and molecular functions respectively.

The top GO directed acyclic graph displayed the GO node (Term) and hierarchical relationship of differentially expressed genes. It involved graphical representation of the results of GO enrichment analysis of DEGs and the inclusion relationship of branches, from top to bottom. The scope of the defined functional descriptions became more specific and prominent with color transition from yellow to red. Top GO acyclic graph for RT vs RC have been provided in supplementary data (Figure S3, S4 and S5).

\section{KEGG enrichment analysis of DEGs}

KEGG pathways gathered information regarding the incorporation of various pathways (plant pathogen interaction, signal transduction and molecular processes). Moreover, pathways which were considerably enhanced in resistant variety were MAPK signaling pathway, plant-pathogen interaction pathway, plant hormone signal transduction pathway, linolenic acid metabolism pathway Isoquinoline alkaloid biosynthesis pathway, phytohormone signal transduction, phenylalanine metabolism pathway, cutin, suberine and wax biosynthesis pathway, and phenylalanine metabolism pathway (Fig. 7A). KEGG enrichment pathways for susceptible cultivar and RT vs ST were also shown in (Fig 7B,7C). DEGs found in various KEGG pathways along with their FC have also been described in Table S3. Results of this analysis indicated that 47, 97,106 and 116 KEGG pathways were embodied under RC vs SC; RT vs RC; RT vs ST; and ST vs SC, respectively. Significant KEGG network path diagrams of resistant variety have been presented in supplementary data (Figure S6, S7, S8). 
Figure 7
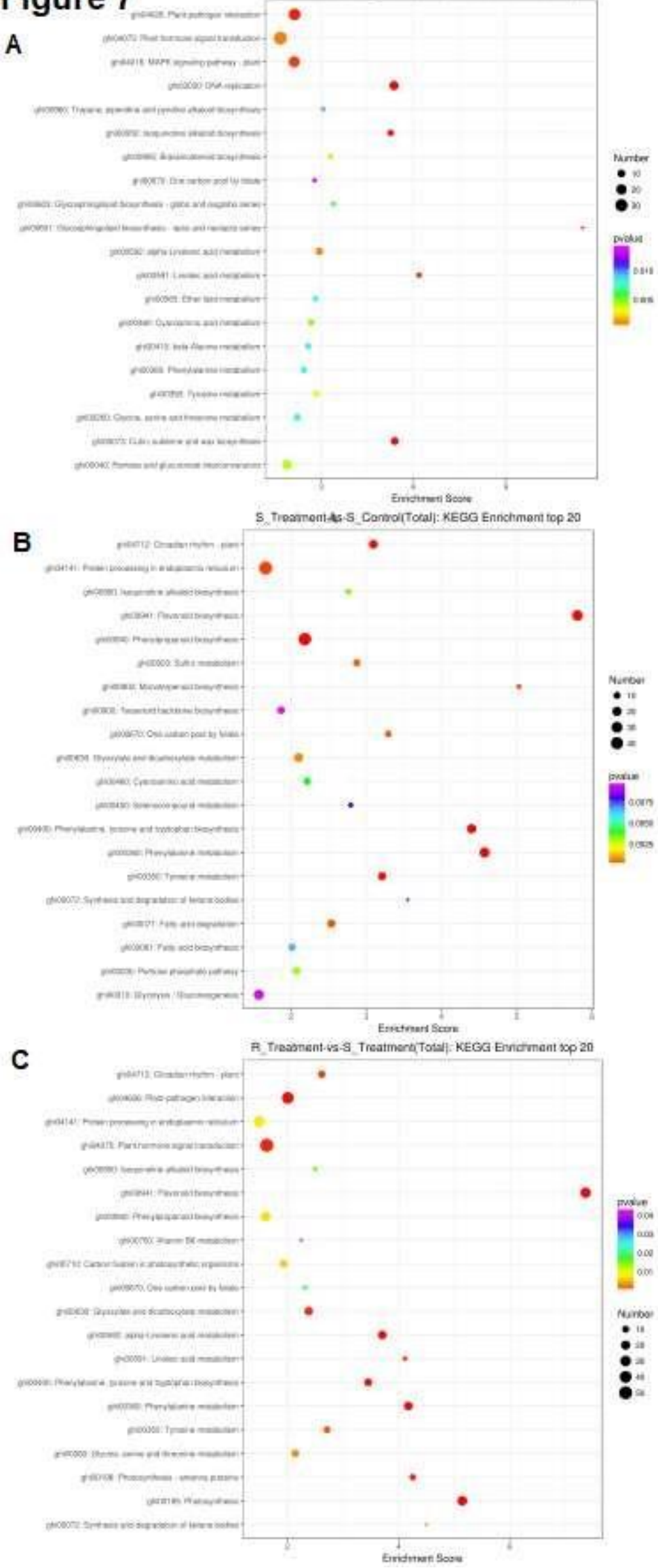
Figure 7. KEGG enrichment top20 bubble diagram of RT vs RC (A)ST Vs SC (B) and RT vs ST (C). The X-axis is the enrichment score. The larger the bubble, the more the number of differential protein-encoding genes contained. The bubble color changes from purple-blue-green-red. With the color transition the scope of protein encoding genes become more well defined. Smaller enrichment $\mathrm{p}$ value represents the greater degree of significance.

\section{Discussion}

Plants use sophisticated constitutive and inducible defense mechanisms to cope with the attacks of pathogens ${ }^{18}$. Different transcriptomic analyses have helped us to understand defense mechanism of cotton plant after the inoculation of $V$. dahliae ${ }^{19}$. Presently, the whole gene profiling of three cotton species (G. hirsutum, G. raimondii and G. arboretum) is accessible ${ }^{20}$. This makes transcription profiling possible and the advancement of RNA-Seq provides insight into the cotton defense mechanism against pathogen infection and also aids in the detection of selected genes, imparting role in disease tolerance.

In the present investigation, defense response of two cotton $(G$. hirsutum) varieties was compared, following the infection of A. tubingensis. Obtained data was adequate for the quantitative analysis of gene expression. Over-all 6,558 genes were differentially expressed.

DEGs involved in "plant hormone signal transduction" and 'plant-pathogen interaction' pathways were considerably enhanced in the leaves of tolerant cotton variety upon fungal inoculation. Consistent with our metabolomics data, in which primary metabolites (sugar, amino acids, fatty acids) and secondary metabolites (alkaloids, terpenoids, pheylpropanoid) exhibited higher accumulation in resistant cultivar ${ }^{17}$.

\section{Genes involved in defense signaling}

In this study, 7 receptor-like protein genes were highly induced in resistant variety post fungal inoculation. In susceptible cultivar, LRR receptor-like serine/threonine-protein kinase $\mathrm{RCH} 1$, cysteine-rich repeat secretory protein 55-like (2 genes) and receptor-like protein 12 (8 genes) were down regulated, post inoculation. These receptors like protein kinases were not expressed in mock inoculated samples. In response to fungal inoculation, nine transcripts encoding mitogen associated protein kinases such as mitogen-activated protein kinase, kinase kinase 3-like, mitogen-activated protein kinase, NPK1-like kinase, mitogen-activated protein kinase, YODA-like kinase, mitogen-activated protein kinase (8-like) were specifically induced in resistant variety. In this study, cellulose synthase like protein (3 genes), caffeic acid 3-Omethyltransferase 1-like (2 
genes), 4-coumarate--CoA ligase-like, and cinnamoyl-CoA reductase 1-like, were upregulated in resistant cultivar, post inoculation.

Plant receptor protein kinases (RPK) illustrate plant pattern-recognition receptors (PRRs) which perceive pathogen signal for prompting an inducible defense ${ }^{21}$. Elevation of cytosolic $\mathrm{Ca}^{2+}$ is one of the prime events in plant intrinsic immunity. Under stress conditions, elevated calcium concentrations are recognized by calcium binding proteins. In plants, wounding and pathogen elicitors activate rapid initiation of Mitogen activated Protein kinase signaling cascade ${ }^{21}$. Instigation of MAPK pathway is one of the earliest studied signaling events after pathogen attack 22. In this study, MAPK signaling cascade was not stimulated in susceptible leaf tissues, upon Aspergillus inoculation. The elicitation of signaling cascade modulates downstream transcription factors, which express the defense related genes and primarily result in improved and persistent defense response. It also provides resistance against invading fungus by directing the generation of antimicrobial proteins and reactive oxygen species ${ }^{10 .}$ NDR1 has been reported to play significant role in defense signaling against pathogens, cell wall adhesion and cell physiology. NDR1 is required for proper activation of the MAPK signal cascade ${ }^{23}$. Specific up regulation of both NDR1 and MAPK cascade in our study shows their probable role in imparting resistance to the tolerant cultivar via signal transduction.

\section{Genes involved in stress response}

In this study, 10 genes of putative disease resistance protein RGA3 were highly induced in resistant cultivar and disease resistance protein RGA2-like was also up regulated in resistant variety. In response to the infection of Phytophthora infestans, resistant genes like TMV and RGA3 have been reported to be upregulated in potato ${ }^{24}$. RPP13 locus of A. thaliana has been described for wide range of resistance against Peronospora parasitica (downy mildew pathogen) ${ }^{25}$. In this study, hypersensitive-induced response protein (1-like) was up regulated in resistant cultivar. Hypersensitive Induced Reaction (HIR) plays role in development of impulsive HR in leaves, following pathogen attacks. During the spontaneous HR lesions, accumulation of development related genes was noticed in plant leaves ${ }^{26}$.

Late embryogenesis abundant (LEA) proteins were found to be expressed in vegetative tissues and these proteins have been reported to be induced by numerous stresses and abscisic acid 27. Among them, calcium dependent signaling triggers LEA-type genes, which then possibly functions in plant defense and repair ${ }^{28}$. Results of current study revealed the up-regulation of three 
genes encoding late embryogenesis abundant protein. At1g64065-like were up regulated in tolerant variety however in susceptible variety, LEA proteins were down regulated. Linoleate $13 \mathrm{~S}$ lipoxygenase 3-1 (chloroplastic-like) genes were highly induced in resistant cultivar while in susceptible cultivar, these were suppressed. In plants, HR response is efficiently activated by LOX genes ${ }^{29}$. In cotton, expression of 9-LOX gene leads to the stimulation of HR-like cell death, in response to the infection of Xanthomonas campestris pv malvacearum. GhLOX1 is linked with the accumulation of salicylic acid during the HR of cotton to X. campestris pv malvacearum ${ }^{30}$.

\section{Genes involved in phytohormone signalling}

In response to pathogen invasion, differential gene expression of phytohormones had been observed in previous studies 5. A. tubingensis inoculation in G. hirsutum resulted in the upregulation of DEGs linked with phytohormone signaling pathways. In the present study, auxin, cytokinin, jasmonic acid, ABA, salicylic acid and gibberellin related genes were differentially up regulated in both varieties, highlighting their roles in plant defense responses. Allene oxide synthase (AOS) gene is involved in translating JA biosynthesis and it was observed to be upregulated in resistant cultivar, following pathogen inoculation. The existence of AOS transcript and JA concentration had been reported to be significantly high in viral and pathogen attacks ${ }^{31 .}$ In the current study, abscisic acid 8'-hydroxylase 1-like (3 genes), auxin-induced protein 22D-like (2 genes), and auxin-responsive protein SAUR6 like (4 genes) were specifically induced in resistant variety, in response to Aspergillus infection. ABA plays significant role in plant defense responses via transcriptional reprogramming of plant cell metabolism ${ }^{32}$. Two genes encoding jasmonic acidamido synthetase (JAR1) and salicylate carboxymethyl transferase-like were upregulated in tolerant cultivar, after fungal inoculation. SA plays a basic role in plant defense and inducing SAR response ${ }^{33}$. JA amino synthetase has been specifically induced in resistant cultivar in post fungal inoculation. JAR1 is a bioactive molecule that converts a JA amino acid synthetase enzyme 34 .

\section{Genes involved in Phenyl propanoid pathway}

Plants accumulate proline-rich glycoproteins, lignins and phytoalexins, as a part of their defense mechanism against fungal pathogen infection ${ }^{35}$. Lignification strengthens plant cell-wall and thus confers resistance to pathogen invasion ${ }^{36}$. In this study, secondary metabolism and phenyl propanoid pathway related DEGs including cellulose synthase like protein (3 genes), 4coumarate-CoA ligase-like, xyloglucan endotransglucosylase/hydrolase protein 22-like, flavonoid 3',5'hydroxylase-like (2 genes), flavonol sulfotransferase-like (2 genes), glucan endo1,3-beta- 
glucosidase 13-like, pectin esterase-like and proline rich receptor like kinases (2 genes) were induced in resistant cultivar, post infection. Genes encoding pectinesterase/ pectinesterase inhibitor (PPE8B-like) were specifically up regulated in resistant variety upon fungal infection.

Studies have shown that pectin methylesterase-inhibiting protein (GhPMEI3) of cotton provides tolerance against $V$. dahliae infection ${ }^{37}$. The phenylpropanoid pathway plays role in plant defense by cell wall fortification, serving as an antioxidant and producting more SA (stress hormone) ${ }^{38}$. The DEGs involved in the phenylpropanoids metabolism exhibited differential expression in both tolerant and susceptible varieties. Phenylalanine ammonia lyase (PAL) was differentially regulated in both cultivars under Aspergillus infection. Genes encoding 4coumarate--CoA ligase 1-like was also up-regulated in susceptible cultivar. The enzymes chalcone synthase (CHS), cinnamoyl-CoA reductase 1-like, cinnamate-4-hydroxylase $(\mathrm{C} 4 \mathrm{H})$, caffeic acid 3-O-methyltransferase-like, and caffeoylshikimate esterase-like were down regulated in susceptible variety after fungal infection. Concurrent up-regulation of genes of phenylpropanoid pathway in response to fungal inoculation endorsed their coordinated production.

Walls are thin 1 (WAT 1) related protein were highly induced in the resistant cultivar while 8 genes of WAT 1 were down regulated in the susceptible cultivar. WAT 1 from Arabidopsis has been studied for its role in the deposition or thickening of secondary cell wall as well as in conferring resistance against vascular pathogens such as Xanthomonas campestris pv. Campestris, Ralstonia solanacearum and V. dahliae ${ }^{39}$. Six genes of cytochrome P450 including 71A1-like (Ghi00905), 86A22 like (Ghi00073), 77A4 like (Ghi00232, Ghi00380, Ghi00591), 84A1ike (Ghi00940) and 82G1-like (Ghi00904) were up regulated in the resistant cultivar.

Studies have shown that numerous CYPs function in phenypropanoid metabolism ${ }^{40}$.

\section{Genes encoding Transcription factors}

Transcription factors (TFs) in plants play part in diverse biological processes including plant innate immunity, pathogen defense response, synthesis of phytoalexin and hormone signaling pathways. In this study, WRKY 7, WRKY 47, WRKY 50, WRKY15, WRKY 53, WRKY46, WRKY 25, WRKY 40, WRKY 41, WRKY 33, and WRKY 57 were specifically induced in resistant cultivar, followed by pathogen inoculation. WRKYs functions in plant defense responses, in addition to various physiological and developmental processes ${ }^{41}$. AtWRKY33 works as a positive regulator of tolerance against Botrytis cinerea and A. brassicicola ${ }^{42}$. In Arabidopsis, AtWRKY53 positively modulates Systemic acquired response. In cotton, GhWRKY33 has been known to be associated with defense response against $V$. dhaliae ${ }^{43}$. WRKY genes, for instance 
GhWRKY11, GhWRKY15, GhWRKY40 and GhWRKY44 have been recognized in regulating plant defense responses ${ }^{44}$. Expression of ethylene responsive $\mathrm{TF}$ is regulated via pytohormones involving SA, JA, ABA, ET and $\mathrm{CK}^{45}$. Specific upregulation of large number of transcription factors in resistant variety depicts its possible involvement in resistance and their down regulation might be the cause of susceptibility of susceptible cultivar.

\section{Genes involved in Oxidative burst}

In plants, generation of reactive oxygen species (ROS) is a hallmark of positive recognition of infection and instigation of defense genes by signaling mechanism. Production of reactive oxygen species has been described under both plant triggered immunity and effector triggered immunity ${ }^{46}$. In tolerant variety, genes encoding glutathione S-transferase (GST) (2 genes) Ghi00480, L ascorbate oxidase (2 genes) and peroxidase (POX) (Ghi00940) were upregulated after fungal inoculation. These genes have been reported to be involved in the processing of ROS. L ascorbate oxidase and GST genes were down regulated in susceptible variety. Glutathione Stransferase is a major scavenger of ROS ${ }^{47}$. POXs (peroxidases) play role in physiological processes including wound healing, lignification, suberization and catabolism of auxin, and defense strategies against pathogen infection ${ }^{48}$. Our results suggested that Aspergillus infection triggered ROS-regulated defense response in leaf tissues of resistant cultivar.

Rationality of our Illumina sequencing data was validated by real time polymerase chain reaction of nine genes having FC 5 or above, under fungal treated condition in both varieties. The comparison of q-PCR expression and fold change values from sequenced data confirmed the credibility of our RNA-seq analysis.

\section{KEGG enrichment analysis of DEGs}

The alkaloid biosynthetic pathways (isoquinoline alkaloid biosynthesis) was highly enriched in resistant cultivar. Expression of various alkaloids is stimulated by fungal elicitors, UV radiation, osmotic shocks and heavy metal ions. The phenyl alanine metabolic pathway was highly enriched in resistant variety and it was also enriched in susceptible variety, in response to Aspergillus infection. The amino acid phenylalanine plays vital role in the interlinkage of primary and secondary metabolism in plants 49 .

In tolerant variety Linolenic acid metabolism pathway was also found to be enriched. Jasmonic acid and its products, produced from alpha-linolenic acid pathway, are primary modulators in plant resistance against necrotrophic pathogens ${ }^{50}$. Up-regulations of sugars, lipids and amino acids metabolisms in plants control signal transduction cascades in plant defense 
strategies ${ }^{(51)}$. Plant hormone signal transduction pathway was also highly enriched in tolerant cultivar. Phytohormones control complicated signaling networks and responses to stresses including biotic and abiotic stresses ${ }^{52}$. MAPK signaling pathway was also specifically enriched in resistant cultivar, post inoculation. By modifying MAPK cascades, plant cells are able to respond to wounding, wide range of stresses and pathogen infections. Importantly, phytohormones are known to effect signaling through MAPK cascades ${ }^{53}$.

Plant hormone signal transduction pathway, plant pathogen interaction pathway, jasmonic acid, salicylic acid. WRKY family and MAPK specifically functions in plant resistance ${ }^{54,55}$. Linolenic acid acts as a precursor of jasmonic acid and intervenes new plant defense signaling pathways ${ }^{56}$. Consequently, it is thought-provoking to inspect DEGs in these three pathway. The pathways involved and enriched under Aspergillus infection in leaf tissues of cotton can be employed for stress resistance. Transcriptome analysis revealed that the genes linked with linolenic acid metabolism, ROS metabolism, phenylalanine metabolism, isoquinoline alkaloid biosynthesis, plant signal transduction and various transcription factors (WRKY, NAC, MAPK) were specifically induced high in resistant cultivar, upon fungal inoculation. Here, our results also offer an essential foundation for better understanding of the defense mechanisms of cotton plant, which in future might assist the breeders.

\section{Materials and Methods}

\section{Preparation of fungus culture and disease assessment}

Strain of A. tubingensis was grown on potato dextrose agar (PDA) at $30{ }^{\circ} \mathrm{C}$ for 6 days. Conidia were harvested by scrapping the mycelium from PDA after adding Czapek broth in the petri plates. The suspension was collected in a sterilized flask and adjusted to our desired concentration $\left(10^{4}\right.$ conidia/ml). Cotton varieties (CIM-573 and NIA-Sadori) were grown in the greenhouse. Greenhouse grown samples were collected in compliance with relevant institutional, national, and international policies and legislation. Leaves of both varieties were used to make small holes and $10 \mu \mathrm{L}$ of the conidial suspension was injected into each hole. Leaves inoculated with only Czapek broth (without fungal conidia) served as mock control. Four days post inoculation (dpi), symptoms were observed and the necrotic areas were measured. Inoculated and mock leaves were collected in liquid nitrogen and stockpiled at $-80^{\circ} \mathrm{C}$ for RNA isolation. Randomized complete block design was employed which comprised of four treatments: RT (resistant variety treated with pathogen), RM (resistant variety with mock inoculation), ST (susceptible variety treated with pathogen) and 
SM (susceptible variety with mock inoculation). For each treatment, four biological replicates were used and entire experiment was repeated three times, over a time interval of 3-4 days.

\section{RNA extraction, library construction and sequencing}

or chemical extraction, greenhouse grown specimens were collected in compliance with relevant institutional, national, and international guidelines and legislation. High quality total RNA of mock inoculated and pathogen treated cotton leaves were extracted using mirVana miRNA Isolation Kit (Ambion $\left.{ }^{\circledR}\right)$. Integrity of extracted RNA was estimated with Agilent 2100 Bioanalyzer (Agilent Technologies, Santa Clara, CA, USA).

Samples having RNA Integrity Number (RIN) $\geq 7$ were preceded for further analysis. The cDNA libraries were constructed using TruSeq Stranded mRNA LT Sample Prep Kit (Illumina, San Diego, CA, USA), according to the manufacturer's instructions. The obtained libraries were sequenced using Illumina sequencing platform (HiSeqTM 2500) and paired end reads (125bp/150 bp) were generated.

\section{Bioinformatics Analysis}

\section{RNA Seq quality assessment and genome mapping}

Raw data (raw reads) was processed and converted to clean reads via Trimmomatic software ${ }^{(57)}$. Raw reads and the obtained results were stored in FASTQ (referred as fq file format), which included sequencing. Reads having poly-N, adopter sequence and low quality reads were eliminated via Trimmomatic, to attain clean reads. By using FastQC, quality of both trimmed and untrimmed reads was assessed. Mapping and alignment of the obtained clean reads to the $G$. hirsutum reference genome was done using HISAT2 aligner ${ }^{58}$.

\section{Gene-Level quantification}

Cufflinks was employed for computing the expression level of protein coding gene (FPKM reads)

${ }^{59}$. Using htseq-count, the read counts of each protein coding gene were attained ${ }^{60}$.

\section{Analysis of differentially expressed genes (DEGs), GO and KEGG enrichment}

Differential expression of genes was evaluated using DESeq. Base mean value was used to determine levels of the expression of gene. The difference multiple was calculated and the significant difference test was performed on the number of reads using NB (negative binomial 
distribution test). For the screening of differentially expressed genes, threshold of $\mathrm{P}$ value (less than 0.05 ) and fold change (more than 2) was set. In order to explore expression pattern of genes, hierarchical cluster analysis was done. To define key biological functions and pathways, all DEGs were mapped to terms in Kyoto Encyclopedia of Genes and Genomes (KEGG: www.kegg.jp/kegg/kegg1.html). Based on the hypergeometric distribution, gene ontology (GO) enrichment and KEGG pathway enrichment analysis of DEGs were correspondingly done, via $\mathrm{R}$ program. Cellular component (CC), molecular function (MF) and biological process (BP) enrichment analysis were performed on the differential genes between samples, using the fisher algorithm and a directed acyclic graph was drawn on the enriched term using topGO ${ }^{61}$. To check the reliability and consistency of biological copies and differences among Aspergillus inoculated and control samples of both varieties, principal component analysis (PCA), hierarchial clustering and correlation among all samples was performed.

\section{Real time PCR analysis}

Using q-PCR, expression of selected candidate genes was determined for the authentication of our transcriptomic data. Ubiquitin was used as a housekeeping gene. Using Primer 3 software tool, forward and reverse primers were designed (Table 2) and synthesized (Sangon Biotech, Shanghai, China). For each sample, three technical replicates were used. Rather than cDNA, RNase-free water was employed as negative control. PCR was done in three replicates in an optical 96-well plate by 7500 Real-Time PCR System (Applied Biosystems), following a standard protocol ${ }^{62}$. PCR mixture was prepared and thermal cycling was executed with an initial denaturation step of $95^{\circ}$ for $1 \mathrm{~min}$, followed by 40 cycles comprising of $95^{\circ} \mathrm{C}$ for $15 \mathrm{~s}, 57^{\circ} \mathrm{C}$ for $15 \mathrm{~s}$ and $72^{\circ} \mathrm{C}$ for $45 \mathrm{~s}$.

\section{Data analysis}

Results were described as mean \pm standard deviation (SD) and investigated by one way analysis of variance (ANOVA, $P<0.05$ ).

Table 2. Primer sequences of selected genes for q-PCR

\begin{tabular}{|l|l|l|l|}
\hline Sr. No & Gene ID & Gene Name & Primers \\
\hline
\end{tabular}




\begin{tabular}{|l|l|l|l|}
\hline 1. & LOC107942464 & $\begin{array}{l}\text { Cytochrome P450 71A1- } \\
\text { like }\end{array}$ & $\begin{array}{l}\text { F TGAGCTTTTCAGCCATTGAC } \\
\text { R AACCGGTGAAGAGAACCAAC }\end{array}$ \\
\hline 2. & LOC107932995 & Peroxidase 45-like & $\begin{array}{l}\text { F TGAGCTTTTCAGCCATTGAC } \\
\text { R TGCAGCAACAGTTGAAATGA }\end{array}$ \\
\hline 3. & LOC107935490 & $\begin{array}{l}\text { WAT1-related protein } \\
\text { At5g07050-like }\end{array}$ & F GAAAGTGAGATGCCAAGCAA \\
R ATGGAGCCTTTGAACCAATC
\end{tabular}

\section{Data availability}

Data are available at http://www.ncbi.nlm.nih.gov/bioproject/751446 of NCBI, accession number PRJNA751446.

\section{References}

1. Bowman, M.J. et al. RNA-Seq Transcriptome Profiling of Upland Cotton (Gossypium hirsutum L.) Root Tissue under Water-Deficit Stress. PLoS One 8, (12) (2013).

2. Chen, Y.et al. Rubisco activase is also a multiple responder to abiotic stresses in rice. PloS One 10, e0140934 (2015).

3. Heidel-Fischer, H. M., Musser, R. O. \& Vogel, H. Annu. Plant Rev. 47, 155-196 (2014). 
4. Naqvi, R.Z.et al. Transcriptomic analysis of cultivated cotton Gossypium hirsutum provides insights into host responses upon whitefly-mediated transmission of cotton leaf curl disease. PLoS One 14, e0210011 (2019).

5. Artico, S.et al.2014. Transcriptome analysis of Gossypium hirsutum flower buds infested by cotton boll weevil (Anthonomus grandis) larvae. BMC Genomics 15, 854 (2014).

6. Zhang, W., Zhang, H., Liu, K., Jian, G., Qi, F. \& Si, N. Large-scale identification of Gossypium hirsutum genes associated with Verticillium dahliae by comparative transcriptomic and reverse genetics analysis. PloS One 12, 8 (2017).

7. Qin, Y.et al. Transcriptome analysis reveals differences in the mechanisms of fiber initiation and elongation between long- and short-fiber cotton (Gossypium hirsutum L.) lines. BMC Genomics 20 ,633 (2019).

8. Bedre, R. Genome-wide Transcriptome Analysis of Cotton (Gossypium hirsutum L.) to Identify Genes in Response to Aspergillus flavus Infection, and Development of RNASeq Data Analysis Pipeline. PloS One 10, 9 (2016).

9. Rajamuthiah, R. \& Mylonakis, E. Effector triggered immunity: Activation of innate immunity in metazoans by bacterial effectors. Virul. 5, 697-702 (2014).

10. Dodds, P. N. \& Rathjen, J.P. Plant immunity: towards an integrated view of plant pathogen interactions. Nat. Rev. Gene 11, 539 (2010).

11. Zhang, S., Du, H. \& Klessig, D. F. Activation of the tobacco SIP kinase by both a cellwallderived carbohydrate elicitor and purified proteinaceous elicitins from Phytophthora spp. Plant Cell 10, 435-449 (1998).

12. Ludwig, A. A., Romeis, T. \& Jones, J. D. CDPK mediated signaling pathways: specificity and cross talk. J. Exp. Bot. 55, 181-188 (2004).

13. De Bruxelles, G. L \& Roberts, M. R. Signals regulating multiple responses to wounding and herbivo1res. Crit. Rev. Plant Sci. 20, 87-521 (2001).

14. Xu, L.et al. Lignin metabolism has a central role in the resistance of cotton to the wilt fungus Verticillium dahliae as revealed by RNA-Seq-dependent transcriptional analysis and histochemistry. J. Exp. Bot. 62, 5607-5621 (2011).

15. Bethke, G.et al. Flg22 regulates the release of an ethylene response factor substrate from MAP kinase 6 in Arabidopsis thaliana via ethylene signaling. Proc. Natl. Aca. Sci. USA 106, 8067- 8072 (2009). 
16. Khizar, M.et al. Aspergillus tubingensis Causes Leaf Spot of Cotton (Gossypium hirsutum L.) in Pakistan. Phyton 89, 103 (2020).

17. Khizar, M.et al. Resistance associated metabolite profiling of Aspergillus leaf spot in cotton through non-targeted metabolomics. PloS one 15, e022867 (2020).

18. 1Bari, R. \& Jones, J. D. Role of plant hormones in plant defence responses. Plant Mol. Biol. .69, 473-488 (2009).

19. Shao, B. X.et al. Analysis of upland cotton (Gossypium hirsutum) response to Verticillium dahliae inoculation by transcriptome sequencing. Genetic Mol Res. 14, 13120-13130 (2015).

20. 2Wang, K.et al. The draft genome of a diploid cotton Gossypium raimondii. Nat. Genet. 44, 1098 (2012).

21. Tena, G., Boudsocq, M. \& Sheen, J. Protein kinase signaling networks in plant innate immunity. Curr. Opin. Plant Biol. 14, 519-529 (2011).

22. Ichimura, K.et al. Mitogen-activated protein kinase cascades in plants: a new nomenclature. Trends Plant Sci. 7, 301-308 (2002).

23. Knepper, C., Savory, E. A. \& Day, B. The role of NDR1 in pathogen perception and plant defense signaling. Plant Signal. Behav .6, 1114-1116 (2011).

24. Yang, X., Tu, L., Zhu, L., Fu, L., Min, L. \& Zhang, X. Expression profile analysis of genes involved in cell wall regeneration during protoplast culture in cotton by suppression 2subtractive hybridization and macroarray. J. Exper. Bot. 59, 3661-3674 (2008).

25. Bittner Eddy, P. D., Crute, I. R., Holub, E. B. \& Beynon, J. L.RPP13 is a simple locus in Arabidopsis thaliana for alleles that specify downy mildew resistance to different avirulence determinants in Peronospora parasitica. Plant J. 21, 177-188 (2000).

26. Zhou, L. et al. Rice hypersensitive induced reaction protein 1 (OsHIR1) associates with plasma membrane and triggers hypersensitive cell death. BMC Plant Biol. 10, 290 (2010).

27. Liu, N.et al. A pectin methylesterase inhibitor enhances resistance to Verticillium wilt. Plant Physiol. 176, 2202-2220 (2018).

28. Xiong, L. \& Zhu, J. K. Molecular and genetic aspects of plant responses to osmotic stress. Plant Cell Envr.25, 131-139 (2002).

29. Maccarrone, M., Melino, G. \& Finazzi-Agro. A. Lipoxygenases and their involvement in programmed cell death. Cell Death Differ. 8, 776 (2001). 
30. Marmey, P. et al. The 9-lipoxygenase GhLOX1 gene is associated with the hypersensitive reaction of cotton Gossypium hirsutum to Xanthomonas campestris pv malvacearum. Plant Physiol. Biochem. 45, 596-606 (2007).

31. 3Alazem, M. \& Lin, N. S. Roles of plant hormones in the regulation of host-virus interactions. Mol. Plant Pathol. 16, 529-540 (2015).

32. Adie, B. A. et al. ABA is an essential signal for plant resistance to pathogens affecting JA biosynthesis and the activation of defenses in Arabidopsis. Plant Cell 19, 1665-1681 (2007).

33. 3Grant, M. \& Lamb, C. Systemic immunity. Curr. Opin. Plant Biol. 9, 414-420 (2006).

34. Thines, B. et al. Browse J. JAZ repressor proteins are targets of the SCF (COI1) complex during jasmonate signalling. Nature 448, 661-665 (2007).

35. Chappe31l, J.\& Hahlbrock, K. Transcription of plant defense genes in response to UV light or fungal elicitor. Nature 311, 76 (1984).

36. Hammond-Kosack, K. E. \& Jones, J. D. Resistance gene-dependent plant defense responses. Plant Cell 8, 1773 (1996).

37. Liu X. et al. GhWRKY25, a group I WRKY gene from cotton, confers differential tolerance to abiotic and biotic stresses in transgenic Nicotiana benthamiana. Protoplasma 253, 1265-1281 (2015).

38. La Camera, S. et al. Metabolic reprogramming in plant innate immunity: the contributions of phenylpropanoid and oxylipin pathways. Immunol. Rev. 198, 267-284 (2007).

39. Denancé, N.et al. Arabidopsis wat1 (walls are thin1) mediated resistance to the bacterial vascular pathogen, Ralstonia solanacearum, is accompanied by cross regulation of salicylic acid and tryptophan metabolism. Plant J. 73, 225-239 (2013).

40. Schilmiller, A. L.et al. Mutations in the cinnamate 4 hydroxylase gene impact metabolism, growth and development in Arabidopsis. Plant J. 60, 771-782 (2009)

41. Jiang, J., Ma, S., Ye, N., Jiang, M., Cao, J. \& Zhang, J. WRKY transcription factors in plant responses to stresses. J. Integr. Plant Biol. 59, 86-10 (2017).

42. Zheng, Z., Qamar, S. A., Chen, Z.\& Mengiste, T. Arabidopsis WRKY33 transcription factor is required for resistance to necrotrophic fungal pathogens. Plant J.48, 592605 (2006). 
43. Zhang, W., Zhang, H., Qi, F. \& Jian, G. 2016. Generation of transcriptome profiling and gene functional analysis in Gossypium hirsutum upon Verticillium dahliae infection. Biochem. Biophys. Res. Commun. 473, 879-885 (2016).

44. Wang, X.et al. GhWRKY40, a multiple stress responsive cotton WRKY gene, plays an important role in the wounding response and enhances susceptibility to Ralstonia solanacearum infection in transgenic Nicotiana benthamiana. PloS One 9, e93577 (2014).

45. Gutterson, N. \& Reuber, T. L. Regulation of disease resistance pathways by AP2/ERF transcription factors. Curr. Opin. Plant Biol. 7, 465-471 (2004).

46. Torres, M. A.ROS in biotic interactions. Physiol. Plant 138, 414-429 (2010).

47. Delprato, M. L., Krapp, A. R. \& Carrillo, N. Green Light to Plant Responses to Pathogens: The Role of Chloroplast Light Dependent Signaling in Biotic Stress. Photochem. Photobiol $91,1004-1011$ (2015).

48. Hiraga, S., Sasaki, K., Ito, H., Ohashi, Y.\& Matsui, H. A large family of class III plant peroxidases. Plant Cell Physiol. 42, $462-468$ (2001).

49. Pascual, M. et al. Biosynthesis and metabolic fate of phenylalanine in conifers. Front. Plant Sci. 7, 1030 (2016).

50. Wasternack, C. Jasmonates: an update on biosynthesis, signal transduction and action in plant stress response, growth and development. Ann. Bot. 100, 681-697 (2007).

51. Rojas, C. M., Senthil-Kumar, M., Tzin, V.\& Mysore, K. Regulation of primary plant metabolism during plant-pathogen interactions and its contribution to plant defense. Front. Plant Sci. 5, 17 (2014).

52. Bari, R. \&Jones, J. D. Role of plant hormones in plant defence responses. Plant Mol. Biol. 69 ,473-488 (2009).

53. Lu, K.et al. Genome-wide survey and expression profile analysis of the mitogenactivated protein kinase (MAPK) gene family in Brassica rapa. PLoS One 10, e0132051 (2015).

54. Birkenbihl, R. P., Diezel, C. \& Somssich, I. E. Arabidopsis WRKY33 is a key transcriptional regulator of hormonal and metabolic responses toward Botrytis cinerea infection. Plant Physiol. 159, 266-285 (2012).

55. Chujo, T.et al. OsWRKY28, a PAMP-responsive transrepressor, negatively regulates innate immune responses in rice against rice blast fungus. Plant Mol. Biol. 82, 23-37 (2013). 
56. Mata-Pérez, C. Transcriptomic profiling of linolenic acid-responsive genes in ROS signaling from RNA-seq data in Arabidopsis. Front. Plant Sci. 6, 122 (2015).

57. Bolger, A. M., Lohse, M \& Usadel, B. Trimmomatic: A flexible trimmer for Illumina sequence data. Bioinform. 30, 2114-2120 (2014).

58. Kim, N. H. \& Hwang, B. K. Pepper heat shock protein 70a interacts with the type III effector AvrBsT and triggers plant cell death and immunity. Plant Physiol. 167, 307322 (2015).

59. Adam, R., Harold, P., Cole, Trapnell. \& Lior, P. Identification of novel transcripts in annotated genomes using RNA-Seq. Bioinform. 27, 2325-2329 (2011).

60. Anders, S. \& Huber, W. Differential expression of RNA-Seq data at the gene level-the DESeq package. Heidelberg, Germany: European Molecular Biology Laboratory (EMBL) (2012)

61. Kanehisa, M.et al. KEGG for linking genomes to life and the environment. Nucleic Acids Res. 3, D480-D484 (2007).

62. Yang, X.et al. Expression profile analysis of genes involved in cell wall regeneration during protoplast culture in cotton by suppression subtractive hybridization and macroarray. J. Exper. Bot. 59, 3661-3674 (2008).

\section{Acknowledgements}

We are thankful to Higher Education Commission (HEC), Pakistan for providing financial support under international research support initiative program (IRSIP-39-BMS-41). We are also grateful to "Shanghai Oe Biotech" (Shanghai, China).

Author Contributions: M.K executed, analyzed and wrote the manuscript. J.S. designed this work and helped in the analysis of results. U.H. assisted pathogen related experiments. M.A. and F.L. helped in statistical and computational analyses. M.A formatted the manuscript. H.S, and A.K. helped in lab experiments. K.M. and H.J.C. prepared graphs and did data analysis. M.F.H.M. supervised the whole experiment.

\section{Figure Legends}

Figure 1. Phenotype of susceptible (A) and resistant cotton cultivar (B), after 4 days of infection with A. tubingensis. (C) Disease severity of susceptible and tolerant cultivars. (D) FPKM density 
distribution reflects the protein-coding gene expression pattern of each sample. (E) The FPKM value box line (F) FPKM box-plot for each sample. The X-axis is the sample name, Y-axis is $\log 10$ $(\mathrm{fpkm}+1)$. The different colors in the figure represent different ranges of fpkm values, the abscissa is the sample, and the ordinate is the number of protein-coding genes.

Figure 2. 3D PCA exhibiting the variance of DEGs in all four data sets, with an overall variance of $74 \%$ contributed by all the three PC.

Figure 3. Statistical representation of differentially expressed genes. The horizontal axis is the comparison group; the vertical axis is the number of differential genes in the comparison group, where "Up" is the number of up-regulated genes with significant differences, and "Down" is the number of down-regulated genes with significant differences. (B) Venn Graph is showing common and unique DEGs between different comparison groups.

Figure 4. Differential expression by volcano map in four data sets. The gray color represents genes with a non-significant difference, while red and green are showing significantly up and down regulated genes. The horizontal axis is the display of $\log 2$ fold change, and the vertical axis is $\log 10 \mathrm{P}$ value.

Figure 5. Relative expressions of candidate genes (DEGs), in fungal inoculated resistant and susceptible varieties where $\mathrm{X}$ axis shows the relative expression of DEGs and $\mathrm{Y}$ axis shows the genes.

Figure.6 GO enrichment analysis of resistant variety (A), Susceptible variety (B) and Resistant pathogen inoculated and susceptible pathogen inoculated (C) where green, blue and red color depicts biological, cellular and molecular functions respectively

Figure 7. KEGG enrichment top20 bubble diagram of RT vs RC(A) ST Vs SC(B) and RT vs ST. The $\mathrm{X}$-axis is the enrichment score. The larger the bubble, the more the number of differential protein-encoding genes contained. The bubble color changes from purple-blue-green -red. With the color transition the scope of protein encoding genes become more well defined. Smaller enrichment $p$ value represents the greater degree of significance.

\section{Conflicts of Interest}

We confirm that all the authors in our manuscript have no conflict of interest. 


\section{Figures}

Figure 1

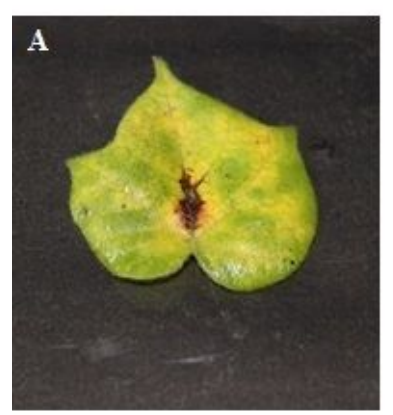

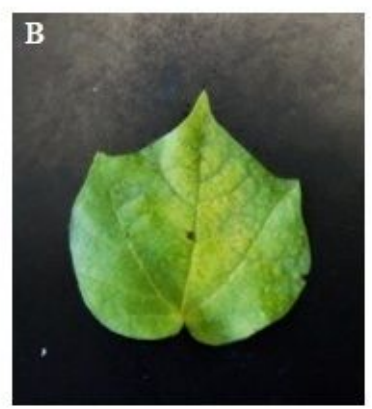

C

Disease Severity

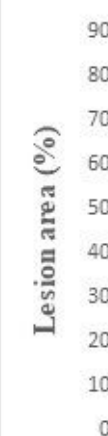
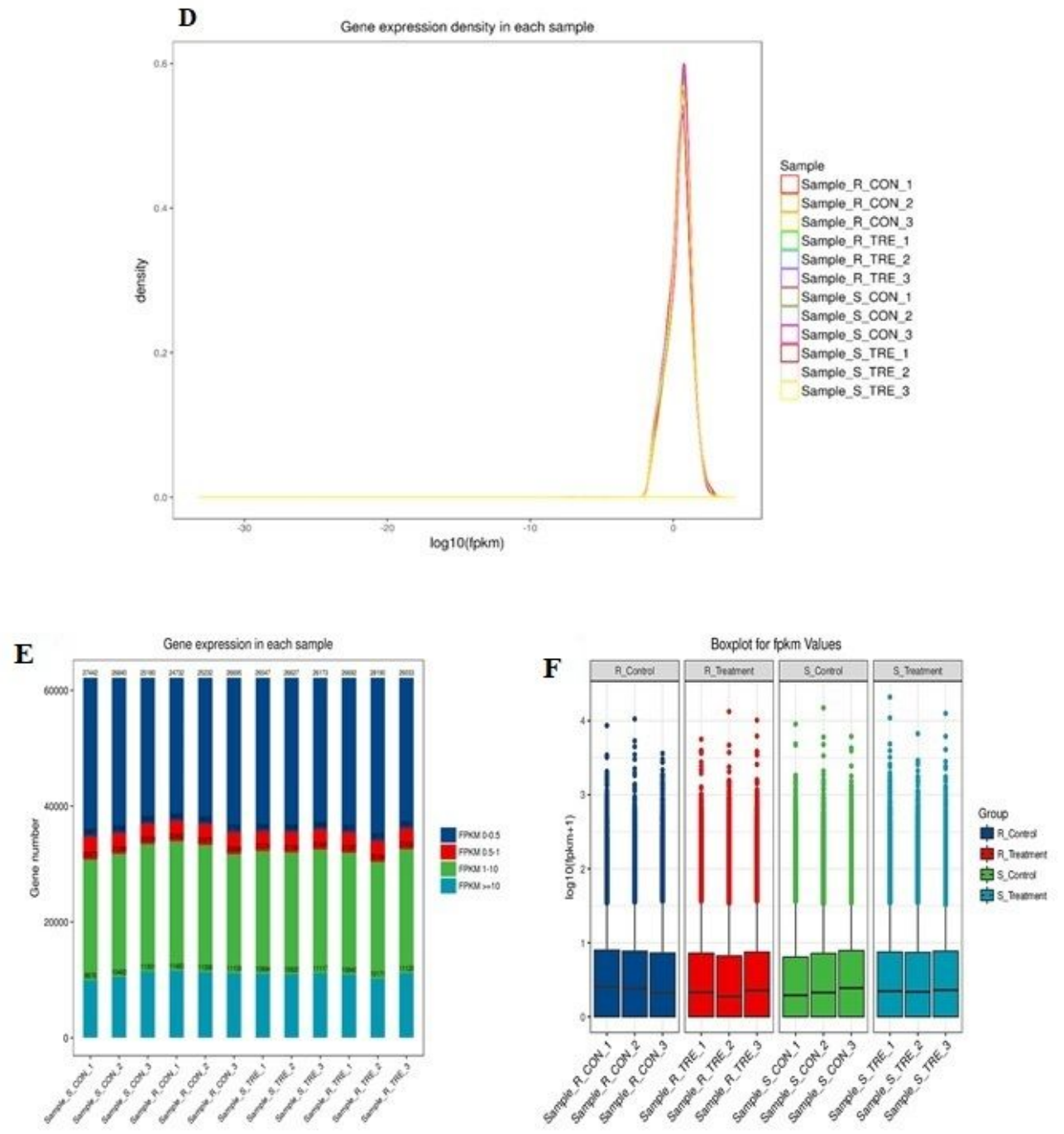

\section{Figure 1}

Phenotype of susceptible (A) and resistant cotton cultivar (B), after 4 days of infection with A. tubingensis. (C) Disease severity of susceptible and tolerant cultivars. (D) FPKM density distribution reflects the protein-coding gene expression pattern of each sample. (E) The FPKM value box line (F) 
FPKM box-plot for each sample. The $\mathrm{X}$-axis is the sample name, $\mathrm{Y}$-axis is $\log 10(\mathrm{fpkm}+1)$. The different colors in the figure represent different ranges of fpkm values, the abscissa is the sample, and the ordinate is the number of protein-coding genes.

Figure 2

PCA 3D figure

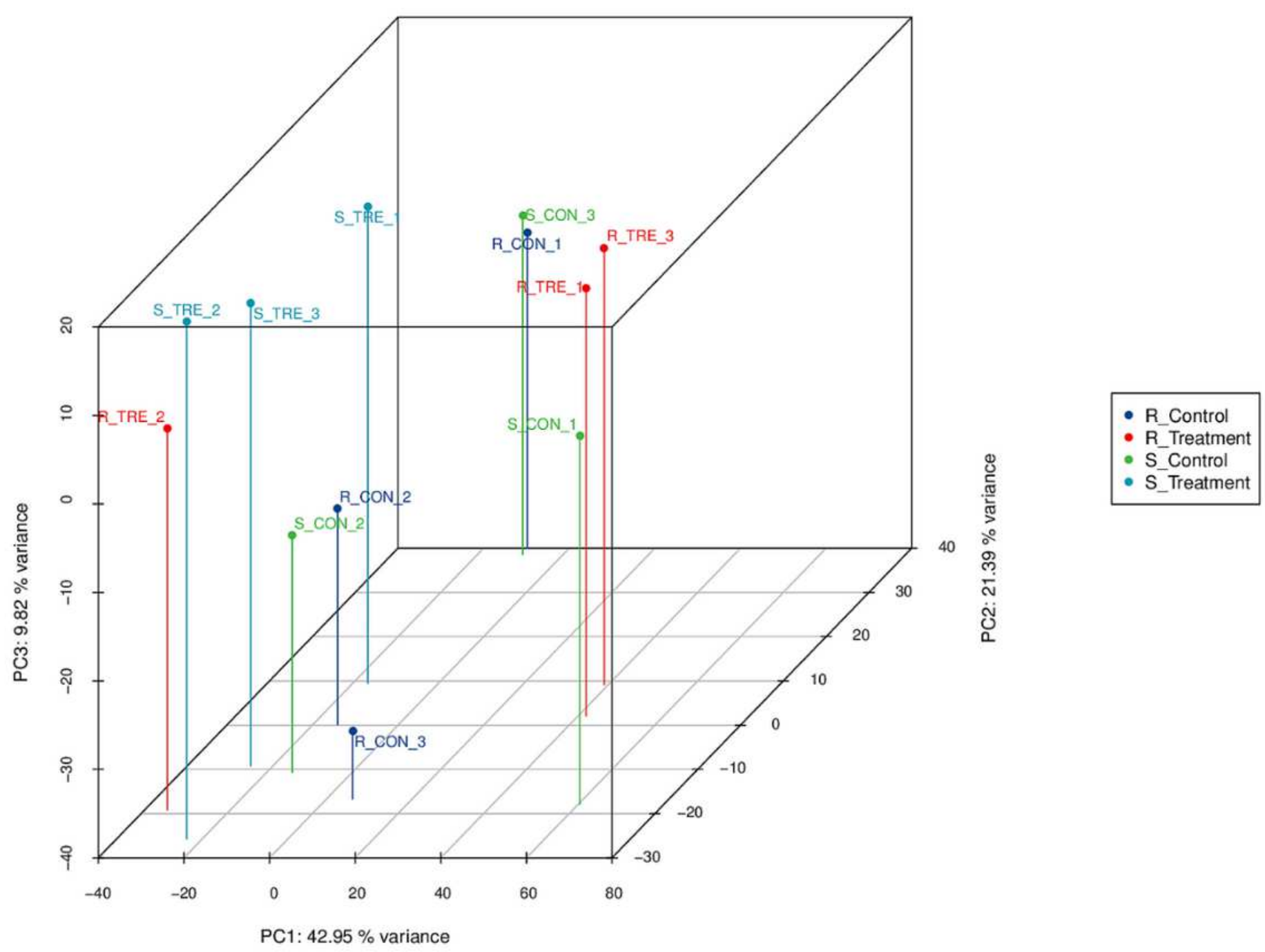

Figure 2

3D PCA exhibiting the variance of DEGs in all four data sets, with an overall variance of $74 \%$ contributed by all the three PC. 
A

Figure 3

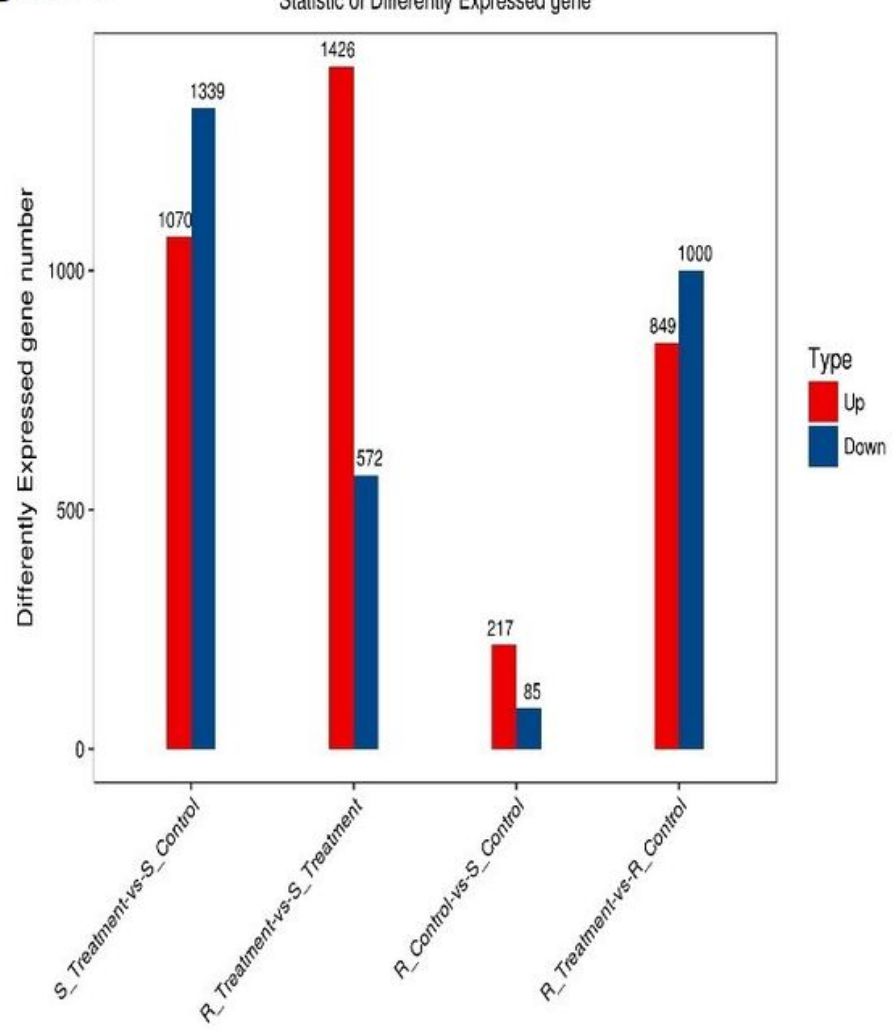

B

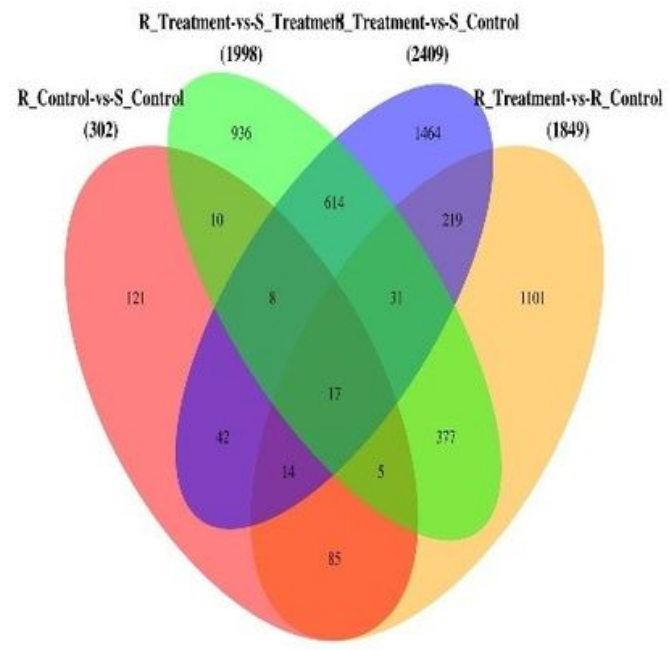

\section{Figure 3}

Statistical representation of differentially expressed genes horizontal .(A) Bar graph in which horizontal axis is the comparison group; the vertical axis is the number of differential genes in the comparison group, where "Up" is the number of up-regulated genes with significant differences, and "Down" is the number of down-regulated genes with significant differences. (B) Venn Graph is showing common and unique DEGs between different comparison groups. 
Figure 4

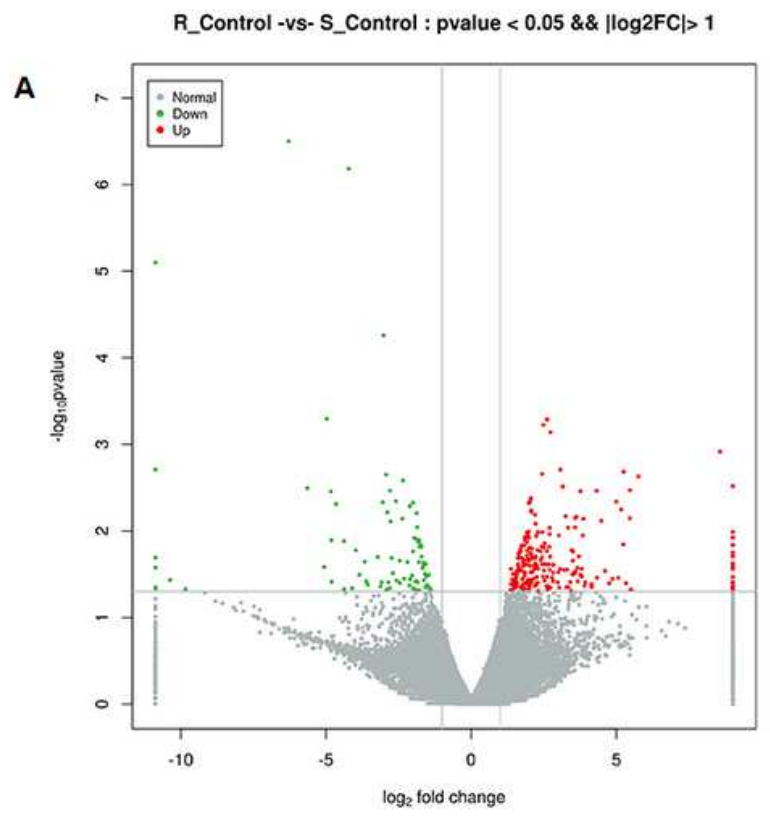

C

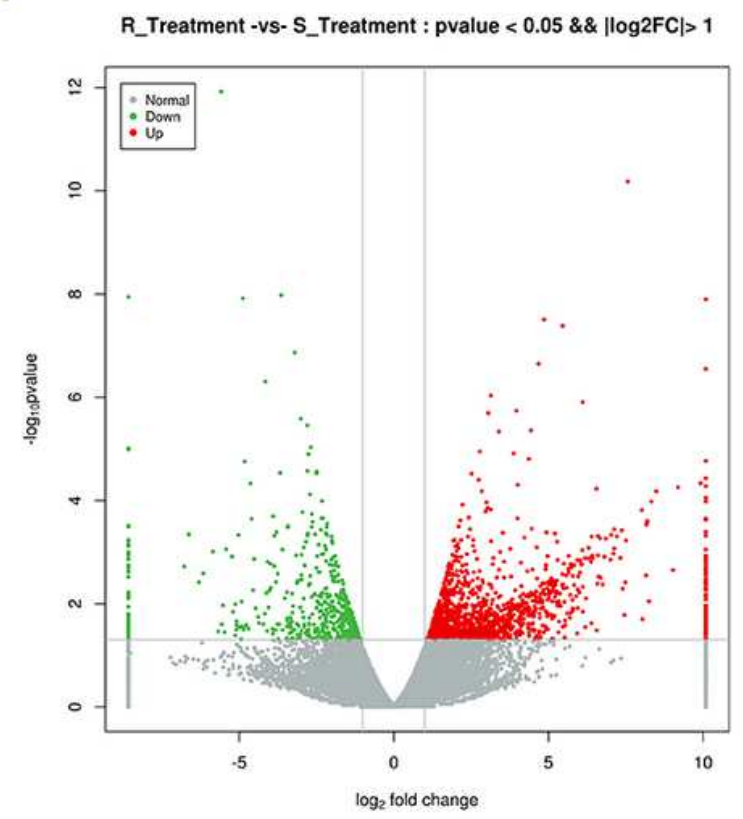

B

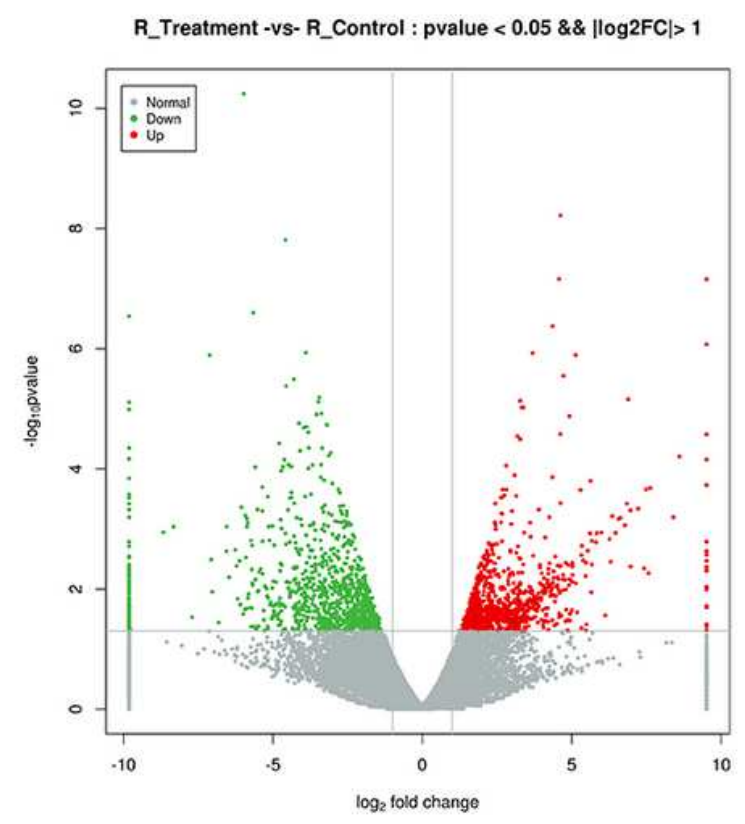

D

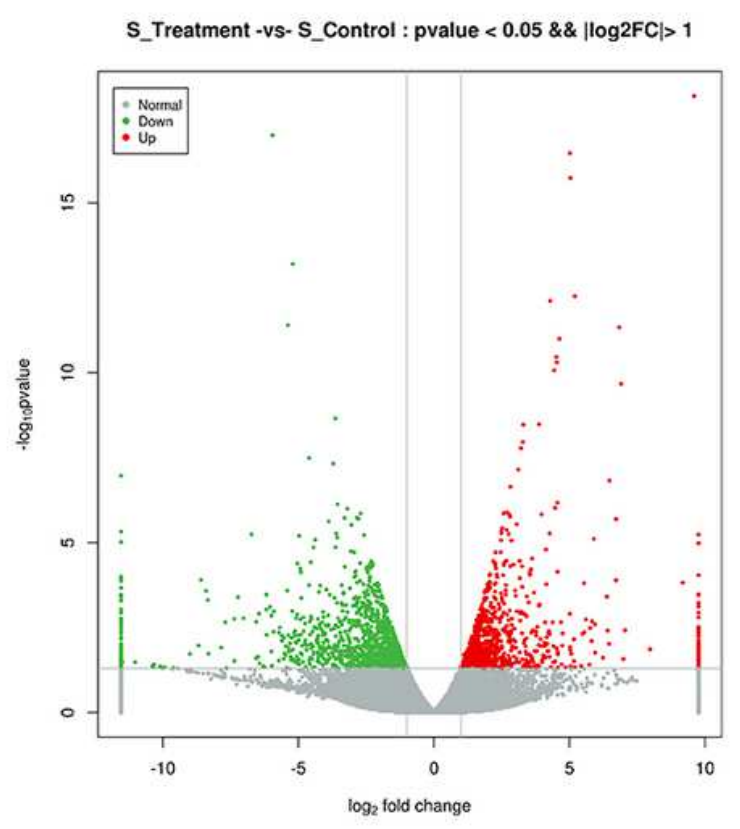

\section{Figure 4}

Differential expression by volcano map in four data sets. The gray color represents genes with a nonsignificant difference, while red and green are showing significantly up and down regulated genes. The horizontal axis is the display of log 2 fold change, and the vertical axis is -log10 P value. 


\section{Figure 5 Expression of Selected DEGs}

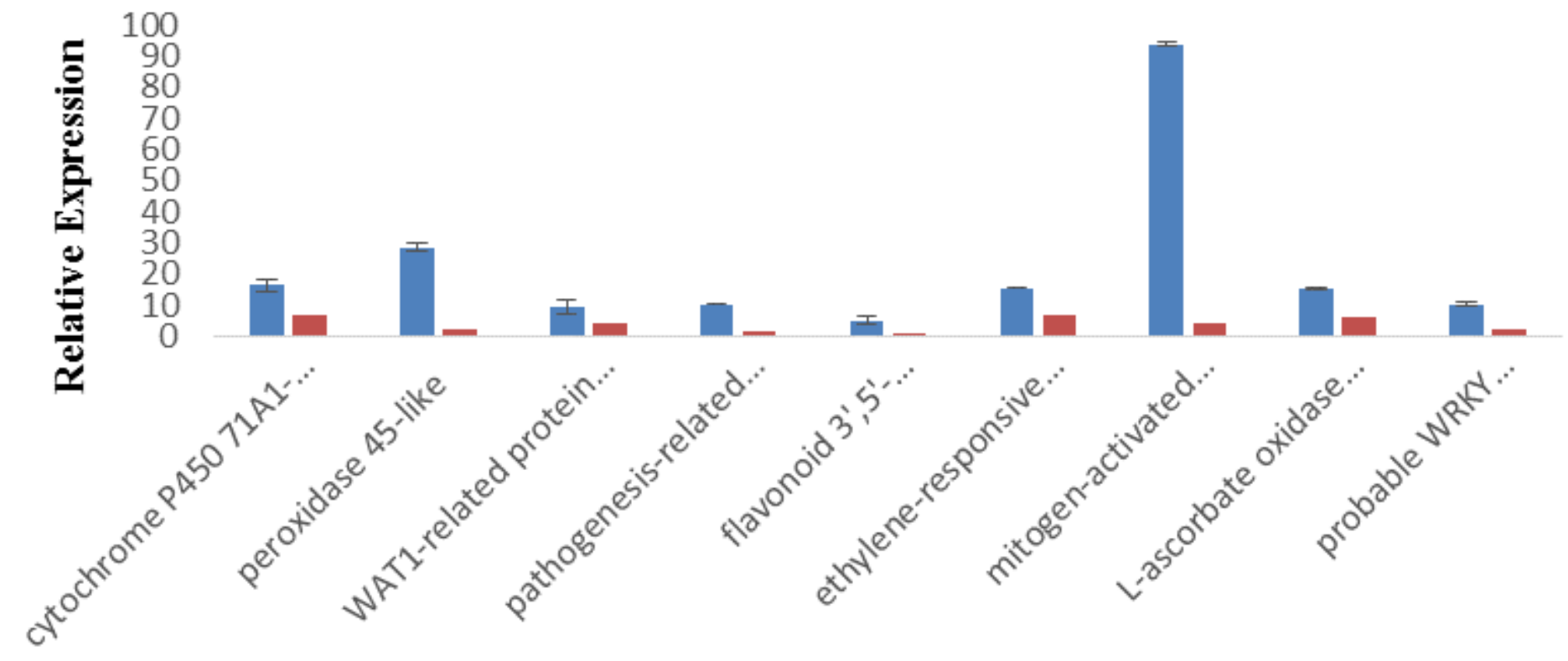

\section{DEGs}

\section{$\square \mathrm{RT} \backsim \mathrm{ST}$}

Figure 5

Relative expressions of candidate genes (DEGs), in fungal inoculated resistant and susceptible varieties where $\mathrm{X}$-axis shows the relative expression of DEGs and $\mathrm{Y}$ - axis shows the genes. 
Fiqure 6

A

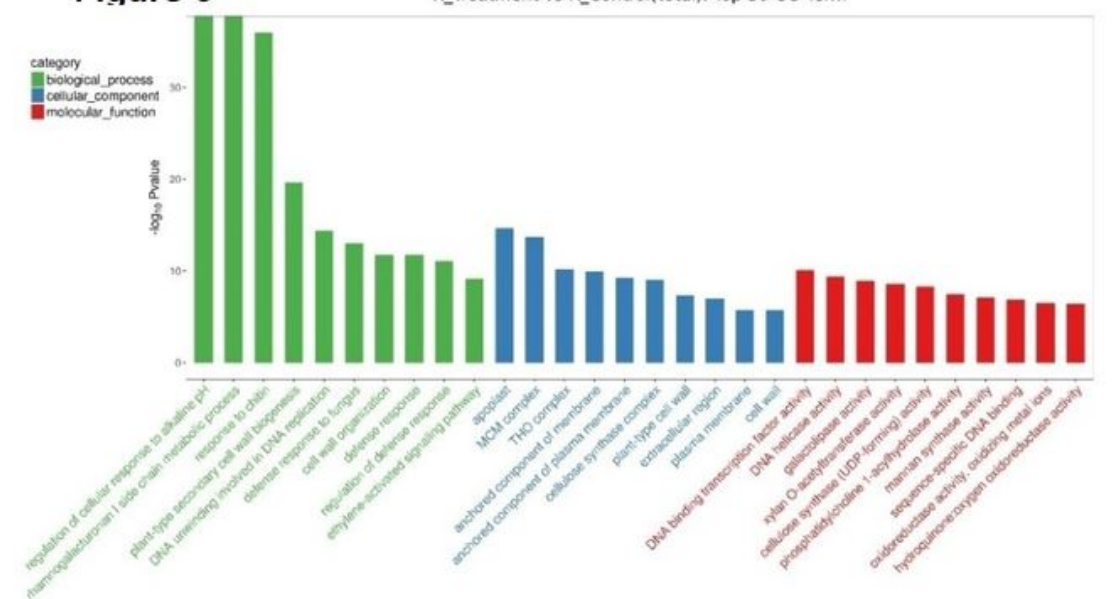

B
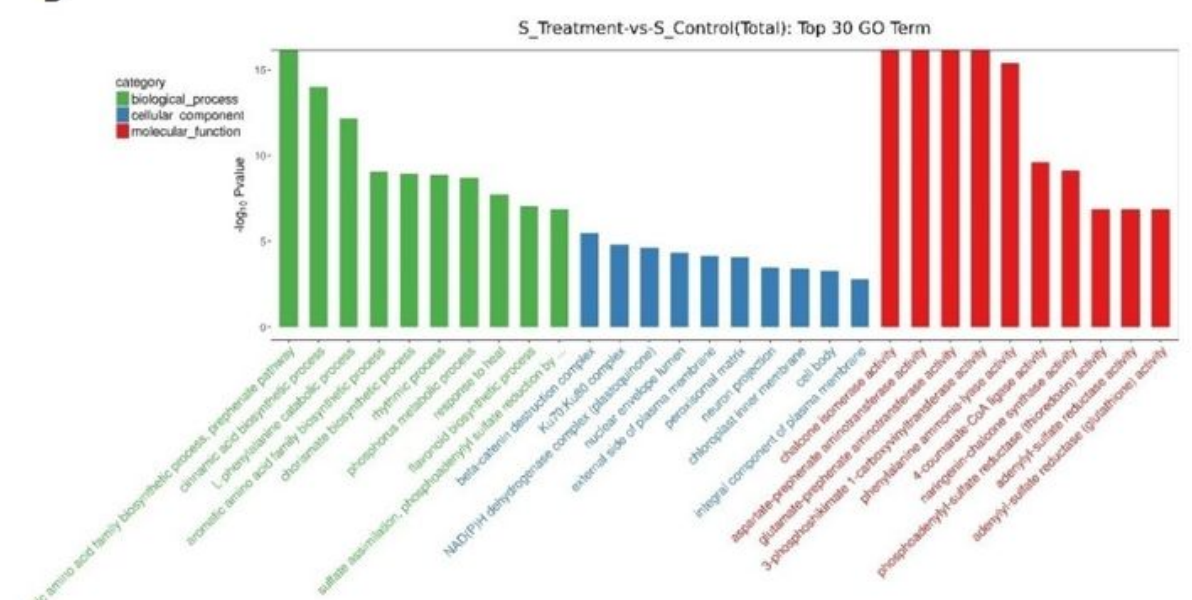

C

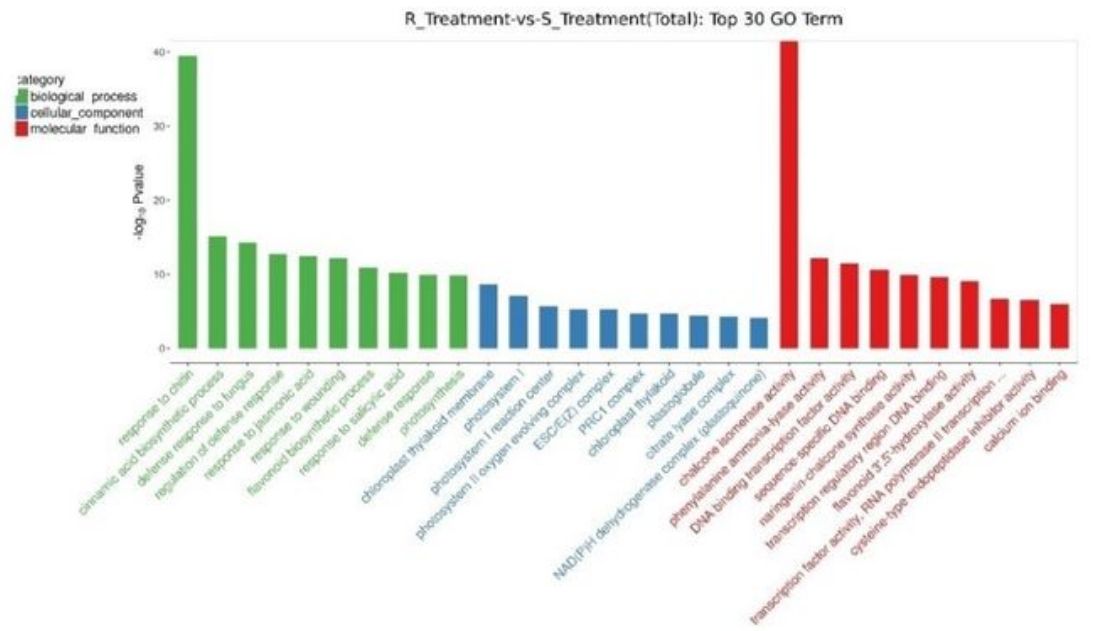

\section{Figure 6}

GO enrichment analysis of resistant variety (A), Susceptible variety (B) and Resistant pathogen inoculated and susceptible pathogen inoculated $(C)$ where green, blue and red color depicts biological, cellular and molecular functions respectively. 

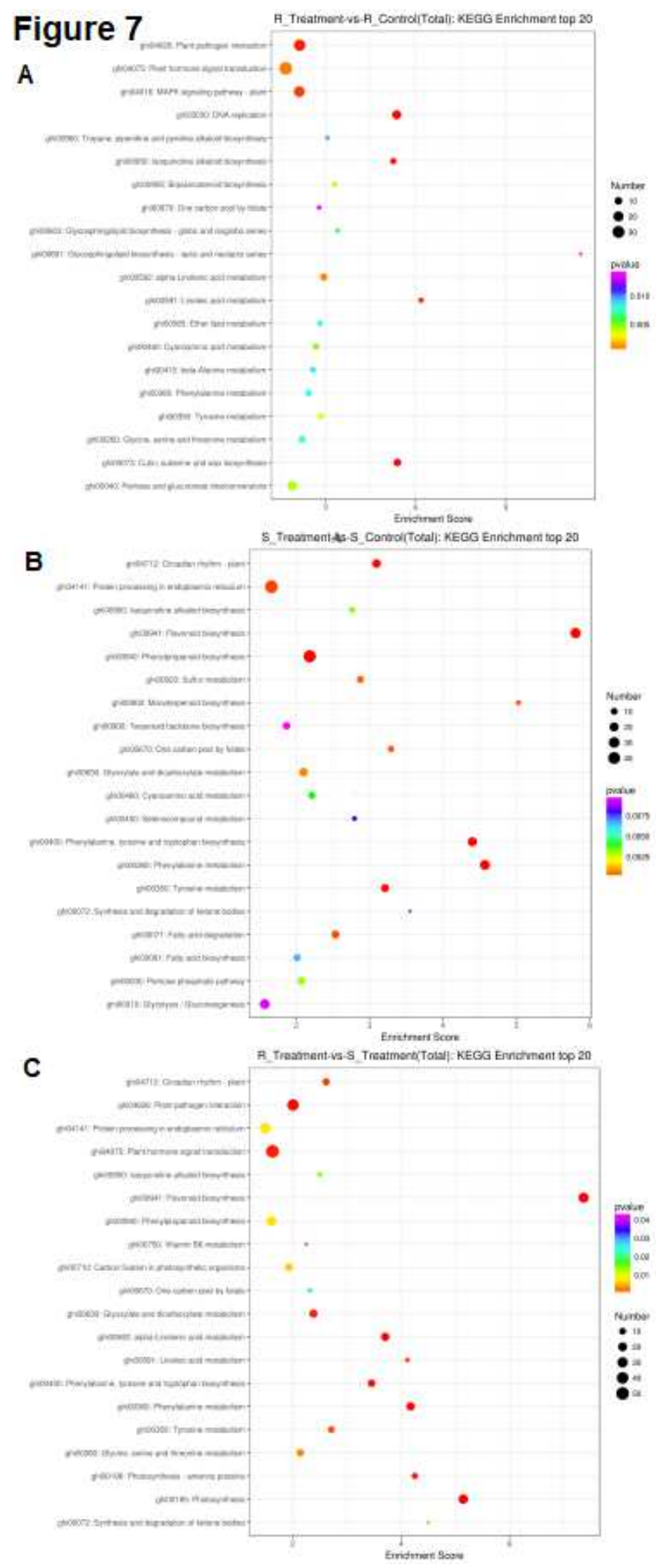

\section{Figure 7}

KEGG enrichment top20 bubble diagram of RT vs RC (A)ST Vs SC (B) and RT vs ST (C). The X-axis is the enrichment score. The larger the bubble, the more the number of differential protein-encoding genes contained. The bubble color changes from purple-blue-green-red. With the color transition the scope of protein encoding genes become more well defined. Smaller enrichment $p$ value represents the greater degree of significance. 


\section{Supplementary Files}

This is a list of supplementary files associated with this preprint. Click to download.

- SupplementaryFiguresfile.docx

- supplementarytable.xlsx 